\title{
Review and Classification of Indices for Voltage Stability Monitoring using PMU Measurements
}

\author{
Sindy L. Ramírez-P*, Carlos A. Lozano-M and Nayiver G. Caicedo-D \\ School of Electrical and Electronic Engineering, Universidad del Valle. Research group in high tension GRALTA, Universidad del Vale, \\ Cali, Colombia.
}

Received 3 April 2018; Accepted 10 October 2018

\begin{abstract}
This paper presents a detailed review of the indices found in the literature for voltage stability monitoring. These indices quantify the condition of stability through a scalar magnitude, which can be monitored by network operators, and take measures to avoid a voltage collapse. Unlike other bibliographic reviews, this paper presents the mathematical formulation of each index and an exhaustive classification is carried out considering the following aspects: if the indices use different types of load, if they are based on phasor measurement units - PMU, if they can be calculated in real time, if they consider the operational constraints that arise in a power electrical system, the test systems in which they have been implemented, and if they have been applied in large power systems. Finally, as a result, an analysis that allows to identify work areas, which have been little explored in this topic, specifically those offered by wide area measurement systems WAMS through PMUs, is presented.
\end{abstract}

Keywords: Phasor measurement unit (PMU), voltage stability monitoring, voltage stability indices, wide area measurement system (WAMS), voltage stability indices.

\section{Introduction}

The results of a voltage stability study provide information on the proximity of the operation point of the Power Electric System (PES) to the point of voltage collapse, known as voltage stability indices, which are scalar magnitudes that vary depending on the system operating conditions.

The complexity of this issue is that in large PES, they contain many electrical variables, have different network configurations, different generation modes according to the dispatch, and contingencies may occur in different elements.

Voltage stability problems generally occur when power systems are overloaded. In many cases, voltage variants are related to abrupt changes due to disturbances that alter the system conditions, such as changes in the topology and/or network setting caused by exit or failure of transmission lines and/or generation units; or in other occasions, due to small changes, such as a load gradual increase. Even though the system is normally designed to put up with these kind of disturbances, abnormal operative conditions could sometimes be originated by contingencies that happen simultaneously, or by unexpected phenomena that put the system under imminent operative danger conditions. Thus, one of the main tasks of network analysts and voltage stability researchers is to study the different phenomena that cause instability, and propose preventive and/or corrective actions when unsafe operative conditions are presented from the voltage perspective [1].

According to the literature review, there are similar works that classify these indices as [2] [3] [4] [5] [6]. In [2]

*E-mail address: sindy.lorena.ramirez@correounivalle.edu.co

ISSN: $1791-2377$ C 2018 Eastern Macedonia and Thrace Institute of Technology. All rights reserved. doi:10.25103/jestr.114.23
[3] [4], voltage stability indices are classified based on the type of information required for their implementation. Indices are divided into two main categories: indices that are based on the Jacobian matrix, and those based on variables and system parameters. In [2], those based on variables and parameters are subdivided on variables obtained by measurements and/or parameters, and on the variables obtained by load flow. The indices based on measurements and/or parameters are grouped into indices considering the Thevenin equivalent, the network equivalent indices, and those based on measurements in general. This paper presents a review until 2012. In [5] and [6], a classification by line and node indices is shown. [5] Also presents the mathematical formulation of the indices, and was carried out until 2015. There are other papers such as [7] [8] [9] [10] [11] [12] that make comparisons between different indexes through simulations made on test systems.

In relation to the indices for voltage stability monitoring based on PMU measurements, in [13] and [14], they are classified into: methods based on local measurements and methods based on Wide Area Monitoring System (WAMS) measurements.

The objective of this article is to classify and review the mathematical formulation of each index in such a way that it is possible to analyze which one of these indices can be implemented in real time, considering real operating conditions of the PES. What it is new in this review is the classification method carried out until 2018, which contains the mathematical formulation of each index, the load models that are considered in the indices, the indices based on PMU measurements, the possibility of real time calculation, the operational constraints of a real PES, the systems used for the implementation of indices, and finally, if they have been applied in large power systems. 
Sindy L. Ramírez-P, Carlos A. Lozano-M and Nayiver G. Caicedo-D/

Journal of Engineering Science and Technology Review 11 (4) (2018) 180 - 198

\section{Theoretical Framework}

A voltage stability analysis determines the operation state, stability limit, stability margin, proximity to instability and critical points of the system, through static and dynamic techniques.

Among the static methods are those based on load flow and sensitivity factors associated with the change in voltage weighted by the change in reactive power (dV/dQ), own values, singular values and Continuous Power Flow method (CPF) [15]. Most of the existing techniques for performing static stability analysis use the solution obtained by the load flow. These techniques provide information on the nature of the problem and the identification of the factors that contribute to instability.

Dynamic analysis is important to complement static analysis and to better understand the phenomenon of instability. The non-linearity of loads and generators is an important factor in determining voltage instability. This type of analysis has the disadvantage of consuming more computational time, due to the requirement of more elaborate models of the elements that make up the PES.

Next, some techniques used, both in the static and dynamic approach, for the analysis of voltage stability are described.

\section{Methods Based on the Jacobian Matrix}

This is one of the most used traditional approaches to perform voltage stability analysis and it is based on the analysis of the mathematical characteristics of the Jacobian matrix or its reduced version $J_{R}$. The latter represents only the linear relationship between the change in the magnitude of bus voltages $\Delta \mathrm{V}$ and the injection of bus reactive power $\Delta \mathrm{Q}$, assuming that there is no variation in the active power $\Delta \mathrm{P}$, as indicated in

$\Delta \mathrm{Q}=\left(\mathrm{J}_{\mathrm{QV}}-\mathrm{J}_{\mathrm{Q} \theta} \mathrm{J}_{\mathrm{P} \theta}^{-1} \mathrm{~J}_{\mathrm{PV}}\right) \Delta \mathrm{V}=\mathrm{J}_{\mathrm{R}} \Delta \mathrm{V}$

These methods provide a measure of the relative proximity between the actual operating point to the voltage collapse point, observing the non-convergence of the load flow, i.e., the singularity of the Jacobian matrix (bifurcation point), when gradual load increments and/or contingencies are performed, such as line outputs and generation units. Within this category, singular value decomposition [16] and modal analysis are found [17].

\section{PV/PQ Curves}

A common practice for the analysis of voltage stability in a power system is the use of PV and QV curves in load nodes under study [15]. The PV curves relate system bus voltages with the active power consumed by the load connected to the node, and similarly for the reactive power by means of QV curves. In general, these curves are obtained by carrying out a large number of power flows using conventional methods. Although this procedure can be automated, it has high computation times and does not provide immediate information to determine the causes of stability problems. Additionally, these procedures focus on individual nodes and its deviations, which could distort the system's stability condition. On the other hand, the nodes for the QV or PV analysis must be carefully chosen, and a large number of curves must be obtained in order to get complete system information. However, it is not possible to fully generate the QV curves due to numerical convergence problems presented by power flow in regions near the point of voltage collapse. There are robust procedures for the determination of PV curves in power systems which use the (CPF). The analysis, by means of a continuous power flow method, employs an iterative process that involves a predictorcorrector scheme in order to find a path of solutions from a base case to the system critical point, which is achieved by including a load parameter under power flow equations [15].

\section{Methods Based on Sensitivities}

This method is based on the analysis of voltage variation with respect to the power in order to determine the margins and limits of voltage stability, as well as the areas and weak zones of the system. It should be noted that the most used sensitivity analysis is the one that relates reactive power to voltage (QV analysis).

The QV sensitivity of a bar represents the slope of the QV curve for a given operating point. A positive QV sensitivity indicates a stable operation, therefore the lower the sensitivity the more stable the system. When stability decreases, the magnitude of sensitivity increases reaching an infinite value in the stability limit. Conversely, a negative QV sensitivity is an indicator of unstable operation; a small negative sensitivity represents a very unstable operation. Due to the non-linear nature of the QV relationship, the magnitude of sensitivities for different system conditions does not provide a direct measure relative to the degree of stability [15], [17].

\section{Monitoring of Reactive Power Reserves}

On-line monitoring of active and reactive power reserves in the system, including generators and other sources of reactive power such as the SVC (Static Var Compensator), have been proposed as indices for voltage safety evaluation.

On the principle that voltage collapse usually does not occur until reactive power limits are reached, especially in large sources of reactive power, operators can be able to determine the proximity of a voltage collapse and, this way, take corrective measures such as load disconnection, based on the additional requirements for reactive power supply in certain areas of the system through the control of the delivery of reactive power and the existing reserves in the system. Reactive power reserves are used to evaluate voltage instability problems in certain areas of the system; these margins are based on reactive power reserves from generators, SVC and synchronous capacitors that deplete the reserves in the process of calculating a QV curve for any node in a given voltage control area [18].

\section{Methods Based on Indices of Voltage Stability}

Voltage stability indices are scalar factors that allow determining the distance from the current operating point to the point of voltage instability as a function of elements, such as the nodal admittance matrix, power flow results as voltage magnitudes or angles, among others. In a complementary way, voltage stability indices allow to detect critical lines and nodes, and define critical areas in voltage stability to apply corrective actions. Currently, considering the increasing use of monitoring devices in PES such as PMUs, it is possible to use real measurements acquired from the PES, which allows the implementation of indices of voltage stability by using online measurements for real-time system monitoring [19].

Many proposals for indices of stability have been made to determine the weakest lines of the system, as well as to identify the weakest nodes or areas in the system. The following section presents a more detailed review of the 
Sindy L. Ramírez-P, Carlos A. Lozano-M and Nayiver G. Caicedo-D/

Journal of Engineering Science and Technology Review 11 (4) (2018) 180 - 198

methodologies used in the definition of indices of voltage stability.

\section{Indices Classification Based on Measurements and/ or Parameters}

In PES it is very important to determine if it is operating within the limits and standards of stability safety, which is achieved through constant monitoring. In regard to voltage stability, numerous methodologies are reported in the literature to measure, with a certain degree of precision, the stability margin of the operating point of the power system. Generally, these measurements have been proposed in the form of Voltage Stability Indices, whose purpose is to determine a scalar magnitude that can be monitored as the system presents changes according to different contingencies, in order to allow operators and network analysts to perform the corresponding preventive and/or corrective actions before a voltage collapse.

In the state of the art of the indices of voltage stability, proposals that provide approximations to define the distance to the point of critical operation in PES are presented. In the classification of these methodologies there are different works, such as [2], [3], [4], and [5], in which voltage stability indices are classified based on the type of information that is required for its implementation. The indices are divided into two main categories as follows: indices that are based on the Jacobian matrix and those based on variables and/or system parameters. The latter is subdivided into variables obtained by measurements and/or parameters system and variables obtained by load flow.

The indices based on the Jacobian matrix, since they are based on the load flow model, have the disadvantage of not considering the dynamic components of the system and, additionally, are not appropriate for an online monitoring scheme.

On the other hand, the indices based on the variables obtained by the solution of load flows have a lower demand of computational time since they are based on the solution of simple equations, facilitating their application in large PES. For the calculation of these methodologies, system parameters such as resistance, and/or reactance of the lines are required, which represents a great disadvantage for its application if there is no real data of the system, which could lead to not very reliable calculations. Additionally, the criterion of the maximum power transfer of an electrical network to loads of power constant type is the most used in indices formulation based on load flows. The above, in some cases, leads to the inaccuracy of the analysis carried out through these indices, due to the great impact that dynamic loads have on a system's voltage stability [2].

In this review, we will adopt the classification made in [2], and we will focus on the methodologies based on measurements and/or system parameters, specifically the indexes based on phasor measurements PMU, which are part of the new technologies of intelligent measurement WAMS (Wide Area Monitoring System) in PES.

The indices based on measurements are intended to use the phasor information of voltages and/or currents, at the point in which the analysis is performed and/or at other PES measurement points, which can provide enough and specific information on voltage instability. PES monitoring has changed in recent years, due to the use of PMUs, which is the most accurate and advanced synchronized time technology currently available. PMUs deliver the measurements of voltage-current phasors and frequency synchronized via GPS [20]. This technology has been reflected in the area of voltage stability in which there are several proposals related to the definition of stability indices using PMUs.

In relation to the indices for voltage stability monitoring based on PMU measurements, several works are reported as in [13] and [14] which are classified into: methods based on local measurements, and methods based on Wide Area Monitoring System (WAMS) measurements. The methods based on local measurements use information delivered by the PMUs in the node where the analysis is being carried out, and, in some cases, they require little or no exchange of information between the monitoring points. These methods accommodate the data time of SCADA and do not require synchronization of time. Most of these methods are based on the adaptation condition of the Thevenin impedance or its extensions, and are based on the assumption that voltage instability is closely related to the maximum load capacity of a transmission network [3]. In contrast, the methods based on WAMS measurements take advantage of the availability of PMUs throughout the PES, which allows having a global view of their behavior. The latter, although having a great advantage over local methods, requires a high number of measurers in order to make the SP fully observable. Finally, some authors classify the indices based on phasor measurements in: methods based on node measurements and methods based on line measurements [5], [6].

Following there is a review of the state of the art of indices for voltage stability monitoring, based on measurements and / or parameters; the review includes the formulation and mathematical definition of each index.

\subsection{Indices Using an Equivalent Model}

These indices consider that the point of maximum transfer of power in a circuit, when considering constant power load, coincides with the starting point of voltage collapse, and this can be reflected in the equality of the impedances (line and load, for a two-node circuit) as shown in Fig. 1. Therefore, some of the indices that are based on this criterion require the estimation of parameters of a network equivalent circuit seen from a load node. In order to establish the parameters of the Thevenin equivalent there are identification algorithms, such as least squares RLS or their variant recursive least squares, which perform the calculations on a sliding window of the discrete data samples, the Transmission Corridor method and the method of approximation proposed in [21], [22] and [3]. These indices can be subdivided into those that use the Thevenin equivalent and those that define their own equivalent [2].

\subsubsection{Indices using a Thevenin Equivalent: Within this category there are the following:}

Chebbo et al., 1992: in [23] an index that does not use estimation algorithms is presented since it obtains the equivalent impedance directly from the $Z$ matrix of the system, using the element of the diagonal $[Z]=[Y]^{-1}$. From the calculation of the magnitude of the relationship between the Thevenin equivalent impedance seen from the load node and the load impedance of the node under analysis, the stability of the node is defined. The formulation of the index is derived from

Fig. 1.

The index to determine the node stability is determined by equation

(2): 
$\frac{\left|Z_{t h}\right|}{\left|\dot{Z}_{L}\right|} \leq 1$

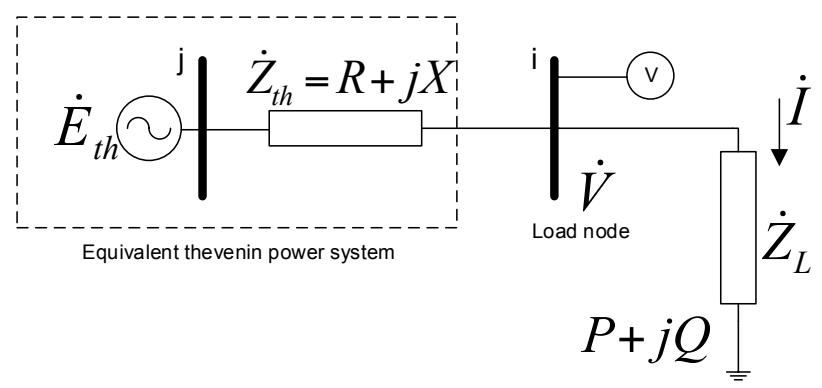

Fig. 1. Two nodes system

Vu et al., 1999: in [24] the Thevenin equivalent estimation is used by equation

(3) by using a recursive least squares algorithm, which requires two measurements in the load node obtained at different and consecutive times.

$\dot{E}_{t h}=\dot{V}+\dot{Z}_{t h} \dot{I}$

Having $\dot{E}_{t h}=E_{t h, R}+j E_{t h, J}$, where R and I denote the real and imaginary part of the corresponding phasors respectively, $\mathrm{V}^{\cdot} \mathrm{I}^{\circ}$ can be obtained from the PMU. When decomposing equation

(3) in the rectangular coordinate system, equation

(4) is obtained:

$\left[\begin{array}{c}E_{t h, R} \\ E_{t h, I}\end{array}\right]=\left[\begin{array}{c}V_{R} \\ V_{I}\end{array}\right]+\left[\begin{array}{cc}I_{R} & -I_{I} \\ I_{I} & I_{R}\end{array}\right]\left[\begin{array}{c}Z_{t h, R} \\ Z_{t h, I}\end{array}\right]$

In order to calculate four unknown parameters of the Thevenin equivalent $\mathrm{E}_{\mathrm{th}, \mathrm{R}}, \mathrm{E}_{\mathrm{th}, \mathrm{I}}, \mathrm{Z}_{\mathrm{th}, \mathrm{R}}, \mathrm{Z}_{\mathrm{th}, \mathrm{I}}$ at least two sets of measurements $\dot{V}$ and $\dot{I}$ are required. If there are more than two sets of $\dot{V}$ and $\dot{I}$ and the measurements are obtained, the Thévenin equivalent parameters can be calculated using the least square method represented by equation

(2).

Julian et al., 2000: In [25], the VIP (Voltage Instability Predictor) index was developed, which also uses the concept of the PES Thevenin equivalent obtained by local measurements in order to define the proximity of collapse in terms of power margin instead of impedance margin. It is evaluated in dynamic regime under conditions of increases of power transfers by using simulations of power flows. To examine the conditions of voltage collapse, the effects of excitation systems and voltage-dependent loads and frequency are considered. The power margin expressed in terms of power is the difference between the maximum power using linear estimation and the current power observed by the VIP voltage predictor which can be expressed by equation

(5):

$\Delta S=\frac{\left(V_{r}-Z_{t h} I_{r}\right)^{2}}{4 Z_{t h}}$

Where, $V_{t}, I_{t}$ are the present voltage measurements and current at the load node, and $Z_{t h}$ is the current Thevening impedance.

When $\Delta S$ is zero, the system is on its maximum power transfer which is the point of voltage collapse.
Milosevic \& Begovic, 2003: in [26], the VSLBI index (Voltage Stability Load Bus Index) is defined. It considers load characteristics (static or dynamic), but it is necessary to know in advance the model and composition of the load, which in most occasions is impossible due to its variance. The VSLBI index is based on consecutive voltage measurements taken in a substation. With them, the comparison between the magnitudes of the load impedance in the substation and the equivalent Thevenin impedance of the system seen from this substation is done by applying the theorem of the maximum power transfer.

The proposed idea is that the voltage phasors contain enough information to detect the voltage stability margin directly from their measurements. When the load is the constant power type, a simple calculation shows that the point of voltage instability coincides with the point of maximum power transfer, producing a relationship between the voltage of an equivalent source $E_{t h}$ and the voltage of a local load node $V$; this way equation

(6) is obtained:

$E_{t h}=2 V \cos (\theta)$

Under maximum transfer conditions, voltage drop $\Delta V$ through transmission impedance $Z_{t h}$ is equal to load node voltage $V_{r}$. Where $\theta$ is the phase angle of voltage $V_{r}$. Therefore, to evaluate the risk of voltage collapse in the presence of a constant load power, it is necessary to monitor the voltage stability index at the load node which is defined as in equation (7):

$V S L B I=\frac{V_{r}}{\Delta V}$

When the system is in conditions of proximity to voltage collapse $V S L B I \cong 1$, otherwise its value is in the range $(1.5$ to 5). The index for a system with $\mathrm{n}$ nodes consists of the lowest value obtained from VSLBI. The VSLBI index, since it is based on the recursive square scheme (RLS) that uses successive measurements to evaluate the Thevenin equivalent, presents some problems which are inherent to the RLS method forgetting factor and the snowball effect. These drawbacks make difficult a robust online application of the method.

Smon et al., 2006: in [27] an alternative to the minimum square method is used, by means of two consecutive voltage and current phasor measurements to formulate the ISI index (Impedance Stability Index). This index uses the Tellegen theorem and attached circuits. The ISI safety index is established from the relationship between two impedances, the load impedance and the Thevenin impedance of the equivalent system, seen from its location point; which are obtained by applying the concept of the attached circuit of the Tellegen theorem.

The ISI index is based on two consecutive voltage and current measures taken in a substation in two moments $t_{k}$ and $t_{k+1}$, from which the comparison between the magnitudes of load impedance in the substation and the equivalent Thevenin impedance of the system seen from this substation is made. For a substation $i$, this index is defined in equation

$I S I=\frac{\dot{Z}_{L}-\dot{Z}_{t h}}{\dot{Z}_{L}}$ 
By substituting the impedances with voltages and currents equation

is achieved:

$$
I S I=1-\frac{|\Delta \dot{V} \dot{I}|}{|\dot{V} \Delta \dot{I}|}
$$

The Thevenin impedance can be easily calculated using equation

$$
\left|\dot{Z}_{t h}\right|=\left|\frac{\Delta \dot{V}}{\Delta \dot{I}}\right|
$$

When having real line measurements, $\Delta \dot{V}$ (voltage differences) and $\Delta \dot{I}$ (current differences) are obtained from the two consecutive measurement samples of a PMU at the node.

Consecutive measurements are taken by PMU measuring devices at the nodes. Under normal operating conditions $I S I \cong 1\left(Z_{K}>Z_{t h}\right)$, and from the point of maximum power transfer, that is to say, in voltage instability $I S I=0$, $\left(Z_{K}=Z_{t h}\right)$.

Nizam et al., 2007: in [28], the PTSI index (Power Transfer Stability Index) uses a hybrid between static and dynamic stability analysis for its formulation. Based on the criterion of maximum power transfer, an index is calculated in each node of the system by using the information of power flow, voltage and Thevenin impedance, and load impedance. The effectiveness of this index depends directly on the parameter estimation algorithm used to determine the Thevenin equivalent [2].

The PTSI index is based on the maximum power transfer limit considering the load as a constant impedance element, thus it predicts voltage collapse in a power system dynamically.

The apparent load power can be written as in equation (11):

$$
S_{L}=\frac{E_{t h}{ }^{2} Z_{L}}{Z_{t h}{ }^{2}+Z_{L}^{2}+2 Z_{t h} Z_{L} \cos (\beta-\alpha)}
$$

Where, $\dot{Z}_{L}$ is the load impedance, $\dot{Z}_{t h}$ is the Thevenin impedance, $\dot{E}_{t h}$ is the Thevenin voltage, $\alpha$ is the phase angle of the load and $\beta$ is the phase angle of the Thevenin impedance.

The apparent load maximum power $S_{L}$ is determined by $\frac{\partial S_{L}}{\partial Z_{L}}=0$, and is equal to equation

(12):

$S_{\text {Lmax }}=\frac{E_{t h}^{2}}{2 Z_{t h}(1+2 \cos (\beta-\alpha))}$

To evaluate the distance from the load node to voltage collapse, a power margin is defined as $S_{L \text { max }}-S_{L}$. The power margin is equal to " 0 " if $Z_{L}=Z_{t h}$, and it means that power can no longer be transferred to that point and voltage collapse occurs. Therefore, voltage collapse occurs when equation presented:

(13) is

$\frac{S_{L}}{S_{L \max }}=\mathbf{1}$

The index PTSI is obtained in equation (14):
$\operatorname{PTSI}=\frac{2 S_{L} Z_{t h}(1+\cos (\beta-\alpha))}{E_{t h}{ }^{2}}$

The PTSI index requires information from the load power, Thevenin voltage and impedance, and load impedance phase angles. The index value is between " 0 " and "1", when PTSI index reaches 1 , meaning that voltage collapse has occurred.

Fu et al., 2008: in [29] index VSI (Voltage Stability Index) is defined with the objective of obtaining an equivalent network model using voltage and current phasor measurements obtained from PMUs; changes are made to the tap of the system reducing transformer, voltage stability indices can be calculated at load supply points. Synchronized voltage and current time measurements, before and after a change of position in the tap, are used to calculate the voltage stability index (VSI) in real time. Fig. 2 shows an OLTC (Transformer On-Load Tap Changer) transformer with tap changer under load at a load supply point. Changing the transformer's position changes voltage on the secondary side of the transformer, thus changing the power flow pattern in the network. By using a PMU to control these two important phasors, the equivalent network model is obtained.

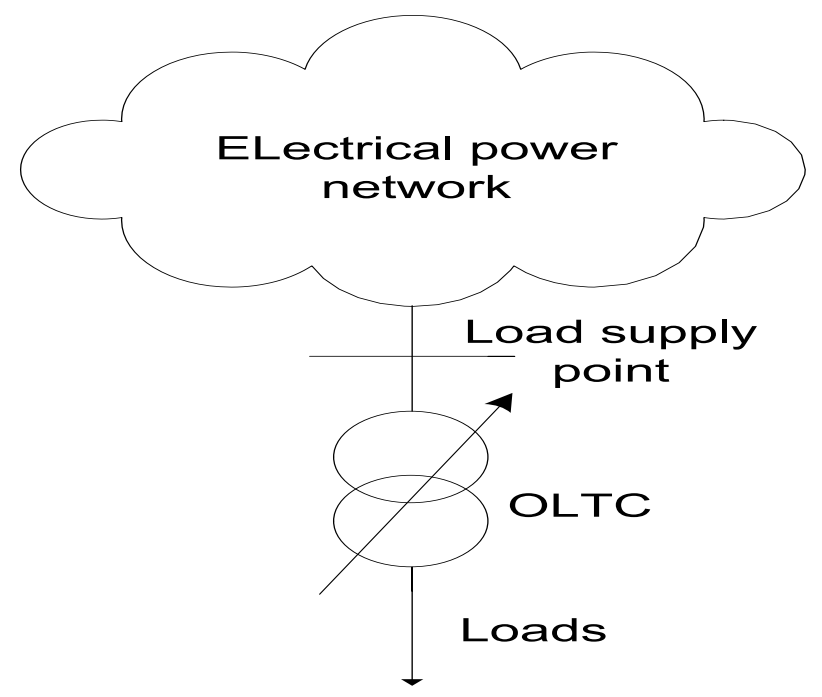

Fig. 2. OLTC installed at load supply points

Considering equation equations

(3), the before and after a tap position change, (16) are obtained:

$\dot{E}_{t h, 1}=\dot{V}_{1}+\dot{Z}_{t h, 1} \dot{I}_{1}$

$\dot{E}_{t h, 2}=\dot{V}_{2}+\dot{Z}_{t h, 2} \dot{I}_{2}$

Where the instantaneous time ' 1 ' is before the tap's movement and ' 2 ' is after the tap's movement. We suppose that $\dot{E}_{t h}$ and $\dot{Z}_{t h}$, do not change during the identification process, which is the moment to move the mechanical parts of the tap changer, usually from 5 to 10 seconds. Equation

(17) is obtained based on

this assumption:

$\dot{E}_{t h, 1}=\dot{E}_{t h, 2}=\frac{\dot{I}_{1} \dot{V}_{2}-\dot{I}_{2} \dot{V}_{1}}{\dot{I}_{1}-\dot{I}_{2}}$

$\dot{Z}_{t h, 1}=\dot{Z}_{t h, 2}=\frac{\dot{V}_{2}-\dot{V}_{1}}{\dot{I}_{1}-\dot{I}_{2}}$ 
After having obtained the values of $\dot{E}_{t h}$ and $\dot{Z}_{t h}$, the PV curve can be plotted assuming the constant $\mathrm{P}-\mathrm{Q}$ relationship or the constant power factor. It is common practice to make such assumptions when plotting the PV curve from which the maximum active power deliverable Pmax can be found due to the following inequality that guarantees a solution for voltage magnitude as illustrated in equation (18):

$E_{t h}^{2}\left(E_{t h}^{2}-4 P R-4 Q X\right)-4(Q R-P X)^{2} \geq 0$

Therefore, a voltage stability index (VSI) for the load supply point can be obtained from equation

(19):

$V S I=\frac{P_{\max }-P_{\text {calculada }}}{P_{\max }}$

Where $P_{\text {calculada }}=\operatorname{Real}\left(\dot{V}_{1} \times \dot{I}_{1}\right)$. From equation (19), a small value for the voltage stability index means that the load feeding point is near the limit of power supply leaving very little voltage stability margin.

Han et al., 2009: in [30] and [31] the WAVI (Wide Area Voltage Stability Index) is presented using the concept of the index shown in [23]. The Thevenin equivalent is obtained with two phasor units located in different areas.

Among the most outstanding characteristics of the proposed index it can be noted that it uses only PMU measurements without coupling with SCA data, it is computationally light to be applied to real-time situation, and it is a new version of the VIP index and adapted transmission corridor for the KEPCO system.

The WAVI algorithm shows whether a power system is stable or not, and uses a similar method to the Z-index VIP.

Fig. 3 shows that the concept of the WAVI method needs at least two PMUs to obtain data from Metropolitan and non-metropolitan areas. The method requires a virtual node in the metropolitan area, and its voltage is the average of all the nodes in the metropolitan area where PMU is installed. A virtual node is also built in the non-metropolitan area. The current that flows from each node will be the sum of the flow of the lines that connect both areas. $\dot{Z}_{S}$ and $\dot{Z}_{T}$ are the shunt and series impedance of transmission lines of the $\mathrm{T}$ equivalent form, respectively.

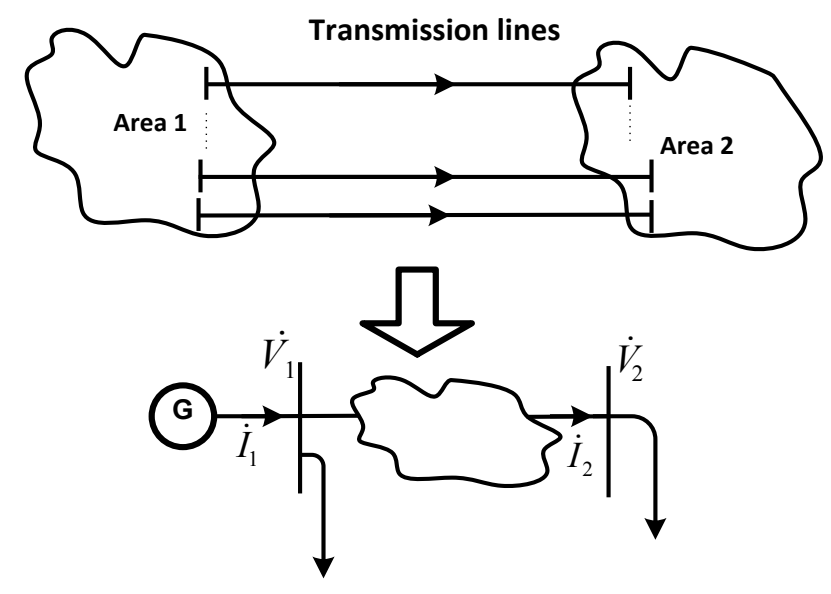

Fig. 3. Wide area system and an equivalent circuit.
To obtain the T-equivalent model of Fig. 4, the Kirchhoff current and voltage laws are applied to obtain the equations. This method requires the calculation of the impedance $\mathrm{Zg}$ of the generators and transformers in the generation area. WAVI uses the VIP method to derive the first $\mathrm{Zg}$ and performs the VIP method by using the virtual node in the non-metropolitan area.

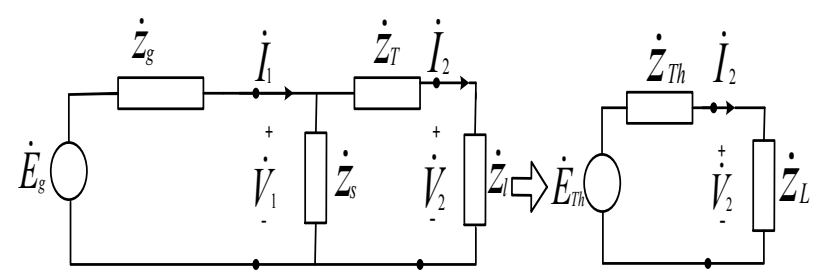

Fig. 4. Reduction to a Thevenin's equivalent

$\dot{Z}_{l}=\frac{\dot{V}_{2}}{\dot{I}_{2}}$

$\dot{Z}_{s}=\frac{\dot{V}_{1}}{\dot{I}_{1}-\dot{I}_{2}}$

$\dot{Z}_{T}=\frac{\dot{V}_{1}-\dot{V}_{2}}{\dot{I}_{2}}$

$\dot{Z}_{t h}=\dot{Z}_{T}+\frac{1}{\frac{1}{\dot{Z}_{g}}+\frac{1}{\dot{Z}_{S}}}$

$\dot{Z}_{L}=\dot{Z}_{l}$

$\dot{E}_{t h}=\dot{V}_{2} \frac{\dot{z}_{t h}+\dot{Z}_{L}}{\dot{Z}_{L}}$

$W A V I=\frac{\dot{Z}_{t h}}{\dot{Z}_{L}}\left\{\begin{array}{c}<1: \text { estabe } \\ \geq 1: \text { inestable }\end{array}\right.$

It is possible to calculate the WAVI index in an area by using only one PMU that provides data per voltage and current phase. If it provides the currents of all the lines that are connected to the installed node of PMUs and the line impedance is known, the voltage and current data per phase of the end of the line can be obtained. Therefore, when the WAVI method is applied to the KEPCO system, only one side of the lines is monitored. Assuming that the line impedances are known, only half the number of PMUs is needed. Equations

(27) and

(28) calculate the voltage and current data per phase at the end of the line.

$V_{2}=V_{1}-\left(\left(\frac{s}{V_{1}}\right)^{*}-j \frac{B}{2} V_{1}\right) \times Z$

$I_{2}=\left(\frac{V_{2}-V_{1}}{Z}+j \frac{B}{2} V_{2}\right)$

$V_{1}$ : Voltage of node $1, I_{1}$ : Current of node $1, V_{2}$ : Voltage of node $2, I_{2}$ : Current of node $2, S$ : Transfer power from node 1 to node $2, Z: \mathrm{R}+\mathrm{jX}$, line impedance and $B$ : line susceptance.

Maharjan \& Kamalasadan.2015. in [35], a voltage stability index to predict voltage collapse in PES is proposed. The index is based on maximum loading capability of the bus combined with an equivalent Thevenin method. From

Fig. 1, solution for voltage $V_{i}$ at node i, is expressed as in equation 
$V S I=\frac{-b \pm \sqrt{b^{2}-4 a c}}{2 a}$

Where,

$a=4 Y_{i i}^{2}\left[\left(P_{i} \cos (\theta)-Q_{i} \sin (\theta)\right)^{2}-\left(P_{i}^{2}+Q_{i}^{2}\right)\right]$

$b=4 V_{j}^{2} Y_{j i}^{2} Y_{i i}\left(P_{i} \cos (\theta)-Q_{i} \sin (\theta)\right)$

$c=V_{j}^{4} Y_{i j}^{4}$

$P_{i}, Q_{i}$ are the active and reactive power of the load, $\theta$ is the angle of the power factor, $Y_{i i}$ the admittance of the load, $Y_{i j}$ the admittance of the line, and $V_{j}$ the sending voltage.

VSI is equal to 1 when the line is reaching its maximum power transfer limit. Once the VSI is less than 1 , the maximum power transfer limit is violated and voltage becomes unstable. The slope of VSI will show the closeness to voltage collapse.

Ramapuram \&Ajjarapu 2017: in [36] and [37] a new voltage stability index based on a Wide-Area Measurement (WAMS), called the Sensitivity based Thevenin Index (STI), is calculated by using the voltage change sensitivity to load variations. The novelty of this index is that it is the first WAMS based method whose value is equal to the Local Thevenin Index (LTI) measured locally at the PMU.

$\mathrm{T}$ he LTI at a load node is the ratio of the estimated Thevenin impedance and load impedance, and it is calculated with equation

using two distinct operating points, with voltage and current phasor differences $\Delta \dot{V}$ y $\Delta \dot{I}$.

$L T I=\frac{\left|\dot{Z}_{t h}\right|}{\left|\dot{Z}_{L}\right|}=\left|\frac{\Delta \dot{V}}{\dot{V}}\right|\left|\frac{\dot{I}}{\Delta \dot{I}}\right|$

The closer the operating points, the better the estimate of accuracy of LTI. However, the closer the operating points are, the higher the chance that measurement noise can adversely impact the estimate. To alleviate this issue, we propose to use the sensitivities to calculate the ideal value of LTI directly, by using a single power flow. The initial load at bus $\mathrm{i}$ is $(P+j Q)$ and the final load is $(P+j Q)(1+\Delta \lambda)$. There is supposed to be a load increase with constant power factor and a loading direction that is proportional to the initial load. The initial load voltage is $(V) e^{j(\theta)}$, and the final load voltage can be expressed as $(V+\Delta V) e^{j(\theta+\Delta \theta)}$.

$L T I=\left|\frac{\Delta \dot{V}}{\dot{V}}\right|\left|\frac{\dot{I}}{\Delta \dot{I}}\right|=\left|\frac{\left(\frac{\Delta V}{V}+1\right)\left((1+\Delta V / V)-e^{-j(\Delta \theta)}\right)}{\left(1+\Delta \lambda-(1+\Delta V / V) e^{-j(\Delta \theta)}\right)}\right|$

\section{Equation (31) is simplified into}

(32), which is rewritten as the STI index

(33). As LTI depends on the $\Delta \lambda$ chosen, it is explicitly written as a function of $\Delta \lambda$, using the expression $\operatorname{LTI}(\Delta \lambda)$.

$L T I=\left|\frac{\frac{\Delta V}{V} \cdot \frac{1}{V}+j\left(\frac{\Delta \theta}{\Delta \lambda}\right)}{\left(1-\frac{\Delta V}{\Delta \lambda} \cdot \frac{1}{V}+j\left(\frac{\Delta \theta}{\Delta \lambda}\right)\right)}\right|$

$S T I=\lim _{\Delta \lambda \rightarrow 0} \operatorname{LTI}(\Delta \lambda)=\sqrt{1+\frac{\left(2 \frac{d V}{d \lambda} \cdot \frac{1}{V}-1\right)}{\left(1-\frac{d V}{d \lambda} \cdot \frac{1}{V}\right)^{2}+\left(\frac{d \theta}{d \lambda}\right)^{2}}}$
Kamel, Karrar, \& Eltom. 2018. in [38], a new normalized voltage stability indicator called the P-index is proposed. It is also shown how the P-index can be used to estimate distance to collapse and the amount of load that is eliminated. Consider the two-bus system shown in

Fig. 1, where the load at node i is $P_{L}+j Q_{L}$ and the voltage magnitude is $\mathrm{V}$. The equivalent load admittance is $G_{L}-B_{L}$, which can be obtained by equation

(34):

$G_{L}=\frac{P_{L}}{V^{2}}, B_{L}=\frac{Q_{L}}{V^{2}}$

The load is gradually increased without a change in its power factor by amounts $\Delta \mathrm{P}, \Delta \mathrm{Q}$, therefore, the corresponding increase in admittance components is $\Delta G_{L}$, $\Delta B_{L}$. The additional load causes voltage magnitude to switch to a quantity $\Delta \mathrm{V}$, which is usually negative; the new node voltage is $\mathrm{V}+\Delta \mathrm{V}$. The increase of active power in the node can be expressed by equation

$\Delta P_{L}=(V+\Delta V)^{2} \Delta G_{L}+(2 V+\Delta V) G_{L} \Delta V$

The P-index is based on the ratio of the two terms, the power lost and the power gained. Additionally, a minus sign is introduced to make the index positive when there is a negative $\Delta \mathrm{V}$, as calculated in equation

(36):

$P-$ index $=-\frac{(2 V+\Delta V) G_{L}}{(V+\Delta V)^{2}} \cdot \frac{\Delta V}{\Delta G_{L}}$

In terms of active power, equation

(37) is obtained:

$P-$ index $=\frac{-2 \frac{P_{L} d V}{V d P_{L}}}{1-2 \frac{P_{L} d V}{V} d P_{L}}$

The index is now defined in terms of normalized voltage and power sensitivities. The stability index has a theoretical value of 1.0 at the stability limit when $\frac{d V}{d P_{L}}=\infty$.

\subsubsection{Indices with own equivalent identification}

This section presents the indices that use an own equivalent network model and the measurements obtained directly from the PES, either locally or globally. Within this category we have:

Hongiie et al., 2005: within the approach that uses an own equivalent, the influence of load model on voltage stability has also been considered. This is how in [39] an improvement was presented to the index L given by [40], through a new index called LI, which considers the influence of ZIP models and exponential load.

Index L can be calculated by using the information from generators with method 1 (equation (

(38)). It can also be calculated by using the information of loads with method 2 (equation (

(39)). Usually, the number of loads is greater than that of generators in a PES, then method 1 is simpler and more popular than method 2. However, when some loads are not of the constant power type, method 2 can give a more reasonable result because it can consider the influence of loads. The LI index uses method 1 to calculate the $\mathrm{L}$ index, and then uses method 2 to adjust this value.

For a n-nodes PES, nodes can be separated into two groups: all load nodes designated as $\sigma_{L}$, and the PV nodes 
located on the tail and denominated $\sigma_{G}$. For any load node $i \in \sigma_{L}$, the voltage stability index $\mathrm{L}$ can be defined by equations

(38) and

(39):

método 1: $L_{i}=\left|1-\frac{\sum_{j \in \sigma_{G}} F_{i j} V_{j}}{V_{i}}\right|$

método 2: $L_{i}=\left|\frac{s_{i}}{Y_{i i} V_{i}^{2}}\right|$

Where, $F_{i j}=-\left|Y_{i i}\right|^{-1}\left|Y_{i j}\right|$ is a complex matrix that defines the relationships between the load voltages and generation nodes, and $Y_{i i}$ is a sub-matrix of $Y_{b u s} . V_{j}$ and $V_{i}$ are the voltages at the generation and load node respectively.

Gong et al., 2006: in [41], a new VSI (Voltage Stability Index) which predicts the voltage stability limit of the PES in stationary state is presented. The maximum load index is based on the criterion of maximum transfer of the PES and uses for its definition the values of maximum active, reactive and apparent powers. This index uses phasor measurements and network parameters to simplify the PES seen from a node by using a network equivalent consisting of a source, a transmission line and a load node.

Given a model of simplified power system as shown in Fig. 1, with time synchronized measurements of voltage magnitude $E_{t h}$, and the load $S=P+j Q$, the maximum transfer of demanded power $P_{\text {max }}$ can be calculated by

(40), where, $\left|\dot{Z}_{t h}\right|=\sqrt{R^{2}+X^{2}}$, assuming that the demand for reactive power $Q$ is constant. Similarly, the maximum reactive power transfer $Q_{\max }$, and the maximum apparent power demand $S_{\text {max }}$, can be calculated by

(41) and (42), assuming that the power demand $\mathrm{P}$, and the power angle of the load $\varphi=\tan ^{-1}\left(\frac{Q}{P}\right)$ are constant.

$P_{\text {max }}=\frac{Q R}{X}-\frac{E_{t h}^{2} R}{2 X^{2}}+\frac{\left|\dot{Z}_{t h}\right| E_{t h} \sqrt{E_{t h}^{2}-4 Q X}}{2 X^{2}}$

$Q_{\max }=\frac{P X}{R}-\frac{E_{t h}^{2} X}{2 R^{2}}+\frac{\left|\dot{Z}_{t h}\right| V_{s} \sqrt{E_{t h}^{2}-4 P R}}{2 R^{2}}$

$S_{\max }=\frac{E_{t h}^{2}\left[\left|\dot{Z}_{t h}\right|-(X \operatorname{sen} \varphi+R \cos \varphi)\right]}{2(X \cos \varphi-R \operatorname{sen} \varphi)^{2}}$

For transmission lines with high $\frac{X}{R}$ ratio, equations approximations leaving aside resistance $R$ are reduced to equations (43),

(44) and

$$
P_{\text {max }}=\sqrt{\frac{E_{t h}^{4}}{4 X^{2}}-Q \frac{E_{t h}^{2}}{X}}
$$

$$
\begin{gathered}
Q_{\text {max }}=\frac{E_{t h}^{2}}{4 X}-\frac{P^{2} X}{E_{t h}^{2}} \\
S_{\text {max }}=\frac{(1-\operatorname{sen} \varphi) E_{t h}^{2}}{2(\cos \varphi)^{2} X}
\end{gathered}
$$

With equations

calculated:
$\boldsymbol{P}_{\text {marg }}=\boldsymbol{P}_{\text {max }}-\boldsymbol{P}$

(46)

$$
\begin{gathered}
Q_{\text {marg }}=Q_{\text {max }}-Q \\
S_{\text {marg }}=S_{\text {max }}-S
\end{gathered}
$$

This method considers that the source voltage magnitude remains constant. The calculation of $P_{\text {margen }}$ is based on the assumption that $Q$ remains constant. Similarly, the calculation of margin $Q_{\text {margen }}$ is based on the assumption that the active power $P$ is constant. The calculation of margin $S_{\text {margen }}$ is based on the assumption that the power factor is preserving the load increase. The margins of $P$ and $Q$ are too optimistic, since normally the demand for active and reactive power increase simultaneously.

The VSI index based on the margins prediction is calculated by using equation

$V S I=\min \left(\frac{P_{\operatorname{marg}}}{P_{\max }}, \frac{Q_{\operatorname{marg}}}{Q_{\max }}, \frac{s_{\operatorname{marg}}}{S_{\max }}\right)$

Small values of VSI indicate that the load node is close to the stable marginal voltage. Once the load node has reached an operating point with stable marginal voltage, its $V S I$ will be equal to zero [41]. In [42], a real application of the VSI index is made in a laboratory.

Genet \& Maun., 2007 y 2009: in [14] and [43], it is assumed that the value of the voltage drop between a load node and the nearest generator can give information that is comparable to the voltage drop through the Thevenin impedance. This new concept overcomes the difficulties encountered in estimating the Thevenin equivalent by means of successive measurements. The VSI index for each node is defined as the relationship between voltage at the load node and the voltage drop at the Thevenin impedance. This new monitoring method based on a wide-area network of Phasor Measurement Units (PMU) detects incipient voltage instability in the PES in real time. In [43], some assumptions are made to approximate the voltage drop. The main assumption is that the generator that is closest to the load can give information comparable to the Thevenin voltage. The distance to a generator is defined as the sum of all the absolute values of the complex voltage drops for each line along the shortest path from a node to the generator. The nearest generator is the one with the minimum electrical distance. If several generators are exactly the same distance, one of them is taken as the closest.

All the generators are not taken into account, only the generators in PV mode that control the active power and the voltage in their output are considered.

The voltage stability index (VSI) is defined for a node $\mathrm{i}$ in equation (50):

$V S I_{i}=\frac{V_{i}}{\Delta V_{i}}$

Where, $V_{i}$ is the voltage at node $\mathrm{i}$ and $\Delta V_{i}$ is the closest distance to the generator. It is assumed that this distance gets close to voltage drop in the Thevenin impedance. If this is accurate, the value of $V S I_{i}$ is one at the point of maximum load capacity. The global VSI index is defined in equation

(51): 


$$
V S I=\operatorname{minVSI} I_{i}
$$

Wang et al., 2009: the ENVCI index (Equivalent Node Voltage Collapse Index) proposed by [44] uses an equivalent PES model seen from the node under analysis; this includes the effects of the local network as well as those of the system that are external to it.

The voltage stability index ENVCI which considers the effect of the rest of the system outside the local network is included through an equivalent model of the system; this ensures the accuracy of the index in modeling. Compared to the internal and external impedance method, the equivalent system impedance, which needs to be estimated by using two states of the system, is only a small part of the total impedance, while the local network impedances (lines that are connected directly to the considered node) are known and do not need to be estimated by using the two states of the system.

The Equivalent System Model (ESM) proposed in [44] is illustrated in Fig. 5:

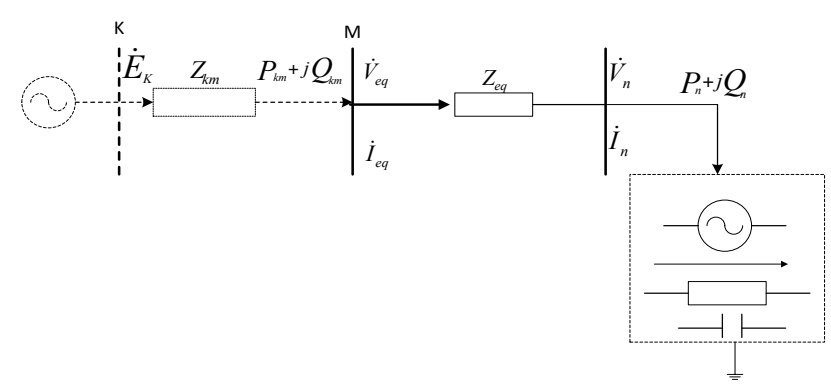

Fig. 5. Equivalent System Model

A made-up voltage source $\dot{E}_{k}$ with impedance $Z_{k m}$ is added to the traditional equivalent local network model to include the effect of the system outside the local network, as shown in Fig. 5. All the ground lines that represent powers of reactive load at the sending ends of the lines with power flows entering node $N$ have been assumed to be part of $Z_{\mathrm{km}}$. $E_{k}$ and $Z_{k m}$, and will have exactly the same effect as the whole external system of the local network, as long as they can guarantee identical voltage phasors and power flows for the equivalent line. To fulfill this purpose, equation

(52) has to be satisfied:

$\left(P_{k m}+j Q_{k m}\right)^{*}=\dot{V}_{e q}^{*}\left(\frac{\dot{E}_{k}-\dot{V}_{e q}}{Z_{k m}}\right)=\dot{V}_{e q}^{*}\left(\frac{\dot{V}_{e q}-\dot{V}_{n}}{z_{e q}}\right)$

Where, $P_{k m}$ and $Q_{k m}$ are the active and reactive powers flowing in the local network. The impedance $Z_{\mathrm{km}}$ represents the impedance of the equivalent system that the power flow finds in the system before it reaches the local network. It is assumed that the equivalent voltage source $\dot{E}^{\prime}{ }_{k}$ and the impedance $Z_{\mathrm{km}}$ are constant between two states of balance of the adjacent system. Superscripts 1 and 2 represent the states of system 1 and 2 as illustrated in equations

$$
\text { (53)and }
$$

$$
\begin{aligned}
& \dot{E}_{k}^{\prime}=K V^{\prime}{ }_{e q 1}+\dot{V}_{n 1}^{\prime}(1-K) \\
& \dot{E}_{k}^{\prime}=K V_{e q 2}^{\prime}+\dot{V}_{n 2}^{\prime}(1-K)
\end{aligned}
$$

(55) and

are obtained:

$$
\begin{aligned}
& K=\frac{1}{\left(1-V^{\prime} e_{e q 1}-V^{\prime} e q 2\right) /\left(\dot{V}^{\prime}{ }_{n 1}-\dot{V}^{\prime}{ }_{n 2}\right)} \\
& Z_{k n}=K Z_{e q}
\end{aligned}
$$

In the previous derivation process, a single line is obtained in which the effects of both the local network and the system outside the local network have been established. By using this model, the two important quantities needed to calculate the new ENVCI can be measured or calculated from the voltage phasor nodes at the two ends of the lines in the second part of the local network. Apparently, the voltage phasor $V_{n} \angle \phi_{n}$ in node $\mathrm{N}$ can be measured directly, while the voltage of the equivalent source $E_{k} \angle \phi_{k}$ can be estimated from voltage phasors and line parameters through the equivalent voltage phasor intermediate $V_{e q} \angle \phi_{e q}$ and the equivalent parameter $Y_{e q}$.

With the equivalent system model of a line for node $\mathrm{N}$ as shown in Fig. 5, the output power in this node has to comply with equation

flow:

(57) of power

$P_{n}+j Q_{n}=\dot{V}_{n}\left(\frac{\dot{E}_{k}-\dot{V}_{n}}{Z_{k m}}\right)^{*}$

Equation (58) provides the voltage stability index ENVCI.

$\mathrm{ENVCI}=2\left(e_{k} e_{n}+f_{k} f_{n}\right)-\left(e_{k}^{2}+f_{k}^{2}\right)$

In polar coordinates, the ENVCI index is presented in equation

$\mathrm{ENVCI}=2 E_{k} V_{n} \cos \phi_{k n}-E_{k}^{2}$

Where, $\phi_{k n}=\phi_{k}-\phi_{n}$

Pérez Londoño et al., 2014: in [45], an improved indicator of the VSI index presented in [14] and [43] is proposed to estimate the voltage stability margin in PES, defined as SVSI (Simplified Voltage Stability Index), which uses voltage measurements and some assumptions from the Thevenin model. The proposed technique uses information about the current operating condition by incorporating only voltage phasor measurements and the topology of the PES. The SVSI index is based on the concept of relative electrical distance, used to select the generator that is closest to a specific load node, and also the association of electrical variables to improve its performance.

The admittance matrix for a PES makes it possible to determine the relationship between current injections and the complex voltages of load (L) and generation (G) nodes, according to equation

$\left[\begin{array}{c}I_{G} \\ I_{L}\end{array}\right]=\left[\begin{array}{ll}Y_{G G} & Y_{G L} \\ Y_{L G} & Y_{L L}\end{array}\right]\left[\begin{array}{c}V_{G} \\ V_{L}\end{array}\right]$

Where $Y_{G G}, Y_{G L}, Y_{L G}$ and $Y_{L L}$ are submatrices of the $Y_{b u s}$ matrix. From equation

equation

obtained:
(60)
Solving equations (54), equations
(53) and 
Sindy L. Ramírez-P, Carlos A. Lozano-M and Nayiver G. Caicedo-D/

Journal of Engineering Science and Technology Review 11 (4) (2018) $180-198$

$\left[\begin{array}{l}V_{L} \\ I_{G}\end{array}\right]=\left[\begin{array}{ll}Z_{L L} & F_{L G} \\ K_{G L} & Y_{G G}\end{array}\right]\left[\begin{array}{c}I_{L} \\ V_{G}\end{array}\right]$

Where, $F_{L G}=-\left|Y_{L L}\right|^{-1}\left|Y_{L G}\right|$ is a complex matrix that defines the relationships between load voltages and generation nodes and can be used to determine the relative distance between these nodes. The elements of the $F_{L G}$ matrix are complex; its columns represent the system generators and each of its rows represent the load nodes. Matrix $D_{L G}$ relates the desired proportion of generation over the programmed load as it is defined in equation

(62):

$D_{L G}=\operatorname{abs}\left[F_{L G}\right]$

The relative electrical distance $R_{L G}$ is obtained from matrix $F_{L G}$ and is given by equation (63):

$R_{L G}=[A]-\operatorname{abs}\left[F_{L G}\right]=[A]-\operatorname{abs}\left(\left|Y_{L L}\right|^{-1}\left|Y_{L G}\right|\right)$

Where $\mathrm{A}$ is a matrix of elements equal to 1 , whose dimensions are $(\mathrm{n}-\mathrm{g}) \times \mathrm{g}$, where $\mathrm{n}$ is the total number of nodes in the system and $g$ is the number of generators. The information given by matrix $R_{L G}$ can be used in place of the route algorithms to obtain the electrical distances between the load and generator nodes.

Once the generator that is closest to a specific load node with matrix $R_{L G}$ is identified, the voltage drop in the Thevenin impedance $\Delta V_{i}$ is estimated by using the following equation:

$\Delta V_{i}=\sum_{b=1}^{n_{j}-1}\left|\dot{V}_{b}-\dot{V}_{b+1}\right| \cong\left|\dot{V}_{g}-\dot{V}_{l}\right|$

Where $\dot{V}_{g}$ and $\dot{V}_{l}$ correspond to the phasor voltages of the nearest generator node and the node analyzed respectively. Equation (64) is a simplification of the one proposed in [14] and [43] because in the new definition of voltage drop only voltage measurements at load and generation nodes are considered.

As the power demand increases, high voltage drops are experienced in some nodes when reaching the point of maximum loadability in one or several nodes of the analyzed power system. This phenomenon indicates a possible voltage collapse, and it is included in the formulation of the SVSI index as the correction factor $\beta$. This factor helps to increase the sensitivity of the proposed index, surpassing the one proposed in [14] and [43]. The correction factor $\beta$ for the SVSI is defined in equation

$\beta=1-\left(\max \left(\left|V_{m}\right|-\left|V_{l}\right|\right)\right)^{2}$

The proposed correction factor is associated with the highest voltage magnitude differences between two nodes, and it can be obtained directly from PMU measurements in the PES analyzed under specific operating conditions. Taking into account the above, the proposed formulation for the SVSI index of node $i$ is given by equation

(66):

$\operatorname{SVSI}_{I}=\frac{\Delta V_{i}}{\beta \cdot V_{i}}$

When the value of SVSI is equal to 1 , it indicates that the point of maximum loadability has been reached (for the type of load being analyzed, this point corresponds to the stability limit). On the contrary, for values close to 0 it is evident that the PES is far from this point, and therefore the system is stable.

Matavalam \& Ajjarapu 2015: in [46], a linear voltage stability index with power demand and based only on angles, called the M-index, is proposed. The linearity of the Mindex can be utilized to directly estimate the load margin. The M-index can estimate voltage stability margin and identify weak buses in a system based on SCADA and PMU data. An equivalent network is used to assess voltage stability. The equivalent model and corresponding voltage phasors are shown in

Fig. 1.

Where $V_{r}$ and $V_{i}$ are the real and imaginary parts of voltage, and $\theta$ is the power factor angle calculated by equation

(67).

$V_{r}=V \cos \delta ; V_{i}=-V \sin \delta ; \tan \theta=Q / P$

The power factor angle $\theta$ is replaced by $(\theta+\emptyset)$, where $\tan \varnothing=\frac{G}{-B}=\frac{R}{X}$ and represents the parameters of the line. The critical angle $\delta_{c r}$, is calculated with equation

(68) and the critical voltage $V_{c r}$, with equation (69):

$\delta_{c r}=\left(45^{\circ}-\frac{(\theta+\varnothing)}{2}\right)$

$V_{c r}=\left(\frac{E}{2 \cos \delta_{c r}}\right)$

An expression for the linear index using the angles aforementioned can be shown in equation

(70).

$M=\left(\frac{\sin \left(\delta_{c r}-\delta\right)}{\sin \delta_{c r}}\right)^{2}$

\subsection{Indices based on Measurements}

In this category there are alternatives to the indices that use equivalents, using the benefits of modern measurement systems. The measurements can be obtained by means of conventional systems such as SCADA, or Wide Area Monitoring Systems WAMS. Some of the voltage stability monitoring methodologies that use global measurements delivered by PMUs are those described in [13], [26] and [47]. For the use of these methodologies a complete observability of the system is required. In order to do this, a large number of installed PMUs are needed which entails an expensive system of implementation; therefore, some researchers prefer the use of local measurements on the system. In the following section some indices that are in this category are cited.

Verbic \& Gubina, 2000: in [48], [49] and [50] the voltage safety index SDI (S Difference Indicator), which analyzes voltage stability by observing what happens with power in each of the transmission lines of a system in the face of events of voltage instability, is presented.

This index is presented again by these authors in articles published in 2003, 2004 and 2006 [47], [51] and [52] with the name of SDC (S Difference Criterion) [53]. SDC is a protection algorithm against voltage instability, which can be implemented in numerical relays using voltage and current phasor measurements at line terminals.

Indices SDI and SDC use consecutive measures of voltage and current taken at one end of a transmission line, with which the apparent power S flowing along the line is measured. For a transmission line connected between the ends $j$ and $i$, the increase in apparent power measured at the 
Sindy L. Ramírez-P, Carlos A. Lozano-M and Nayiver G. Caicedo-D/

Journal of Engineering Science and Technology Review 11 (4) (2018) 180 - 198

end $i$, after two consecutive measurements $k$ and $(k+1)$, is expressed as the sum of two phasors $F 1$ and $F 2$ as presented in equation:

$\Delta \dot{\mathrm{S}}=\Delta \dot{V}_{i} \dot{I}_{i j}^{*}+\dot{V}_{i} \Delta \dot{I}_{i j}^{*}=F_{1}+F_{2}$

The increase in apparent power is zero when the measured variables do not change significantly. This occurs in $\Delta \dot{V}_{i}=0$ and $\Delta \dot{I}_{i j}^{*}=0$, which corresponds to the case of normal operation and when $m=\frac{\left|F_{1}\right|}{\left|F_{2}\right|}=1$ and $\partial=$ $\left|\Delta F_{1}-\Delta F_{2}\right|=\pi$; this way, the index SDI is defined in equation

$\mathrm{SDI}=1+m \cos (\partial)$

For a system with $\mathrm{n}$ transmission lines, the index is defined as:

$\operatorname{SDI}_{\text {sistema }}=\min \left(S D I_{n}\right) \forall u=1, \ldots, n$

Dividing equation equation

(74) is obtained:

$\Delta \dot{\mathrm{S}}=1+\frac{\Delta \dot{V}_{i} i_{i j}^{*}}{\dot{V}_{i} \Delta \dot{i}_{i j}^{*}}=1+a e^{j \partial}$

Now, the increase in apparent power is zero when: 1) $\Delta \dot{V}_{i}=0$ and $\Delta I_{i j}^{*}=0$, and 2) $\alpha=1$ and $\partial= \pm \pi$. This way, the index SDC is defined through equation

(75):

$\mathrm{SDC}=\left|1+\frac{\Delta \dot{V}_{i} i_{i j}^{*}}{\dot{V}_{i} \Delta \dot{i}_{i j}^{*}}\right|=\left|1+a e^{j \partial}\right|$

Under normal operating conditions $\mathrm{SDC} \approx 1$, and at the point of voltage collapse $\mathrm{SDC} \approx 0$.

The main problem with these indices is that they require to verify that the line is loaded below its nominal load. This method presents good results for load increases, but in more realistic cases parameters do not get to be adjusted to obtain useful results. Strong oscillations are observed when one of the generator limiters acts. This is explained by the fact that the method implicitly assumes that the Thevenin equivalent parameters do not vary between two sampling points. When a generator is limited and is no longer a source of voltage, the parameters of the Thevenin model are modified considerably and the assumption is not correct [43].

Kim et al., 2007: in [54] an index called VSRI (Voltage Stability Risk Index) is defined, which identifies the risk of PES before a voltage collapse for each node of the system by using time series of RMS voltages at the system load nodes. VSRI does not require any input from the other nodes for its calculation; therefore, this algorithm can be integrated in the PMU, located in different nodes to evaluate system voltage stability in transient state. The moving average of the time series data over a particular window $\mathrm{j}$ is calculated using measurements in $\mathrm{N}$ at previous time instants $i$.

$v_{j}^{m a}=\frac{\sum_{i=1}^{N} y_{i}}{N}$

Using the moving average $v_{j}$ in equation (76), the percentage of diversity of the $i$-th measurement is calculated through equation

(77): $d_{j}=\frac{v_{i}-v_{j}^{m a}}{v_{j}^{m a}} \times 100$

Index VSRI is defined in equation

(78):

$z_{j}=\frac{\sum_{i=1}^{N}\left(d_{j}+d_{j-1}\right) \Delta t}{2 N}$

Where $v_{i}$ is the voltaje measurement at the ith time instant, $v_{j}^{m a}$ is the moving average of window $j, z_{j}$ is the index VSRI of window $j, \mathrm{~N}$ represents the PMU measurements available, and $\Delta t$ is the subinterval in the integral trapezoidal rule.

Venkatesh et al., 2007: with the apogee of alternative energies, in [55] the index called DVCI (Dynamic Voltage Collapse Indicator) is presented and determined for real time. It calculates the maximum apparent power flow margin of the feeder that connects the wind generator to the distribution system, using local measurements of power and voltage.

DVCI index is derived from the transmission line model of a distribution system as shown in Fig. 6. Shunt capacitors are ignored in this model since voltage levels in the distribution systems are low and line lengths are short.

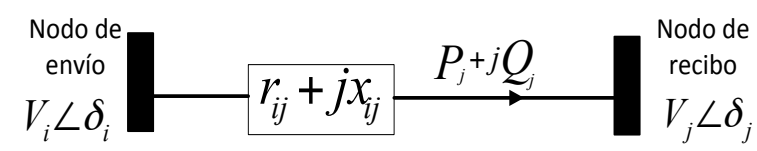

Fig. 6. Model of a transmission line in a system with any number of nodes.

An expression for the maximum loadability index (MLI) in terms of voltage at the emitter end $V_{i}$ and power at the receiver end $\left(P_{j}+Q_{j}\right)$ is presented in equation (79). The term MLI when multiplied by $\left(P_{j}+Q_{j}\right)$, results in a measure of the maximum power that can be received at the receiver end of the transmission line for a sending voltage $V_{i}$

$M L I=\frac{V_{i}^{2}\left[-\left(r_{i j} P_{j}+x_{i j} Q_{j}\right)+\sqrt{\left(r_{i j}^{2}+x_{i j}^{2}\right)\left(P_{j}^{2}+Q_{j}^{2}\right)}\right]}{2\left(x_{i j} P_{j}-r_{i j} Q_{j}\right)^{2}}=f 1\left(V_{i}, P_{j}, Q_{j}\right)$

MLI is greater than 1.0 and MLI is equal to 1.0 when $\left(P_{j}+Q_{j}\right)$ is equal to the maximum load of the line. The final power at the receiver end can be related to the final power of delivery as in equation (80):

$P_{j}+Q_{j}=\left(P_{i}+j Q_{i}\right)-\left(r_{i j}+j x_{i j}\right)\left|I_{i}\right|^{2}=f 2\left(P_{i}+j Q_{i}\right)$

Where $I_{i}$ refers to the sending current. Therefore, (79) can be modified using equation (80) to obtain an expression of the dynamic voltage collapse indicator (equal to MLI) only in terms of sending parameters as shown in equation $(81)$ :

$D V C I=f 1\left(V_{i}, P_{j}, Q_{j}\right)=f 1\left(V_{i}, f 2\left(P_{i}+j Q_{i}\right)\right)=f\left(V_{i}, P_{i}, Q_{i}\right)$

The term in (81) can be calculated by a micro processed relay to identify if a feeder of the distribution system is close to voltage collapse. Finally, the DVCI index only uses locally measurable parameters at the emitter end.

Haque., 2007: in [56], a simple VSI (Voltage Stability Index) method is proposed to determine the distance to 
voltage collapse by using little local information, such as voltage and current magnitudes. The VSI varies almost linearly with the load and is used to estimate the distance to voltage collapse by using linear extrapolation. The effects of generator reactive power limits, line output, non-uniform load increase and shunt reactive power sources on the VSI were also investigated.

For a load node $i$ the index VSI proposed in [56] is calculated by equation

$V S I_{i}=\left[1+\left(\frac{I_{i}}{V_{i}}\right)\left(\frac{\Delta V_{i}}{\Delta I_{i}}\right)\right]^{\alpha}$

Where, $V_{i}$ and $I_{i}$ are the voltage and current magnitudes of the load node, $\Delta V_{i}$ and $\Delta I_{i}$ are the changes in voltage and current in the face of changes in the load apparent power. In general, when the load of a node increases the current also increases, and the voltage of the load decreases. $V S I_{i}$ is zero at the point of voltage collapse. $\alpha$ is a constant number equal or greater than 1 , which is used to give a linear characteristic to the index.

Smon et., 2008: in [57], an improvement is made to the SDC index since the new BSDC (Bus apparent-power difference criterion) index uses load currents instead of line currents in a node, and therefore, there is no additional verification to see if the lines are generating or consuming reactive power. This index is based on the measurement of the apparent power delivered to the node, instead of the one that is flowing along the line. At the receiver end of a line $j$ $i$, which supplies a node $n$ as in Fig. 7, $\dot{V}_{n}$ is the voltage of node $n$, which is supplied by current $\dot{I}_{n j}$.

The point of voltage collapse is when $\Delta \dot{S}_{n}=0$. In the BSDC index the load current and the apparent power delivered to the node as presented in equations

(83),

and

considered:

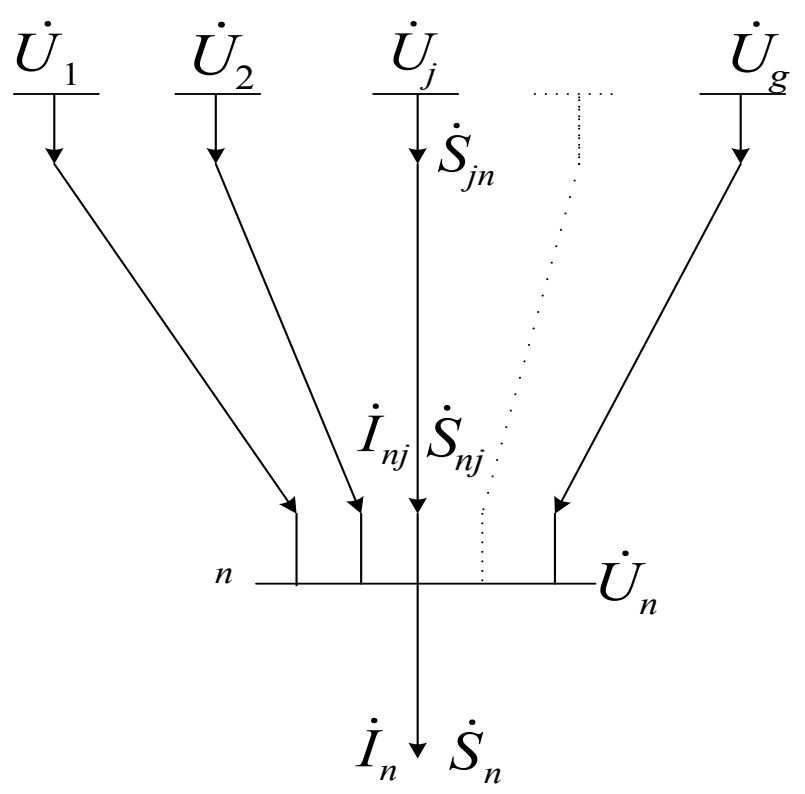

Fig. 7. Supply to the load node n.

$$
\begin{aligned}
& \Delta \dot{S}_{n}=\Delta \dot{V}_{n} \dot{I}_{n}^{*}+\dot{V}_{n} \Delta \dot{I}_{n}^{*}=0 \\
& \left|\dot{V}_{n} \Delta \dot{I}_{n}^{*}\right|=\left|-\Delta \dot{V}_{n} \dot{I}_{n}^{*}\right|
\end{aligned}
$$

$\left|\frac{\dot{V}_{n}}{\dot{I}_{n}^{*}}\right|=\left|\frac{\Delta \dot{V}_{n}}{\Delta \dot{i}_{n}^{*}}\right|$

Where $\Delta \dot{V}_{n}$ and $\Delta \dot{I}_{n}^{*}$ represent the complex voltage and current increases for node $n$ with respect to their base-case values $\dot{V}_{n}$ and $\dot{I}_{n}^{*}$. . The increments are determined after the system is subjected to a series of disturbances in the system, with two consecutive online measurement samples of the node voltage and the load current of a PMU at node $n$. The BSDC index is defined in equation

(86):

$B S D C=1-\left|\frac{\Delta \dot{V}_{n} \dot{i}_{n}^{*}}{\Delta \dot{i}_{n}^{*} \dot{V}_{n}}\right|$

Arya et al., 2008: in [58], the Li (Line Voltage Stability Index) for type 21 relays is presented. The process to obtain the index is based on the fact that before a load increase, the impedance seen by an impedance relay becomes equal to the line impedance at the point of collapse for the most critical line. Power flow equations have been used to identify critical lines, which may be vulnerable during higher loadability conditions.

Considering a transmission line, with ABCD constants known as an integral part of the PES, it is assumed that the line is connected between nodes ' $s$ ' and ' $r$ ' as shown in Fig. 8 . Furthermore, it is assumed that the actual power flow direction is from node 's' to node ' $r$ '.

Power flow equations at the receiver end ' $r$ ' are written in equations $\quad(87)$ and (88):

$$
\begin{aligned}
& p_{r}=A v^{2} \cos (\beta-\alpha)-v \cos \left(\delta_{r}-\delta_{s}+\beta\right) \\
& q_{r}=A v^{2} \sin (\beta-\alpha)-v \sin \left(\delta_{r}-\delta_{s}+\beta\right)
\end{aligned}
$$

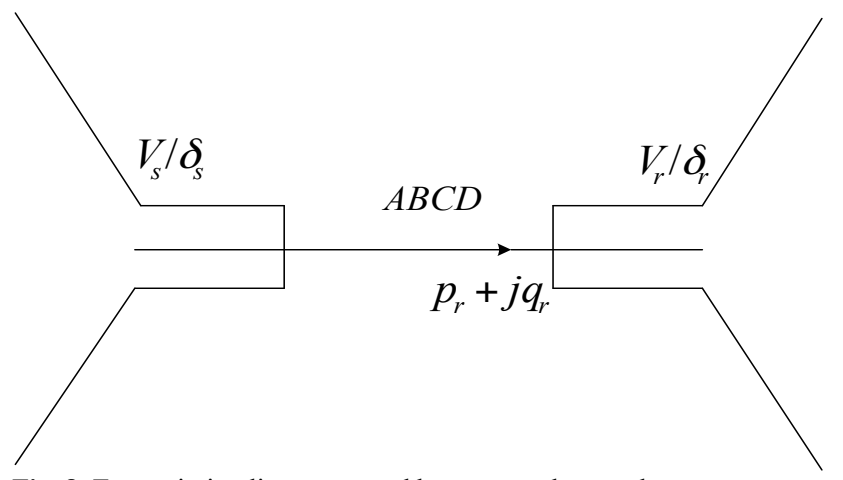

Fig. 8. Transmission line connected between nodes s and r.

In previous equations $v=V_{r} / V_{s}, P_{N}=V_{s}^{2} / B, p_{r}=$ $P_{r} / P_{N}, q_{r}=Q_{r} / P_{N}$, where $P_{r}$ and $Q_{r}$ are the power flow equations at the receiving end. The line voltage stability index $\mathrm{Li}$ is obtained with equation

$L_{i}=A v \cos (\delta-\alpha)$

$\delta=\delta_{s}-\delta_{r}$

$\alpha$ is the angle of the parameter $\mathrm{A}$ of the line; this parameter can be calculated with equation

(92). As the system gets stressed, the index is reduced from $A \cos \alpha(\cong 1)$ to 0.5 at the point of collapse. In an interconnected system one of the lines will be critical; in fact, lines can be classified according to the magnitudes of this line voltage stability index. The line voltage stability 
Sindy L. Ramírez-P, Carlos A. Lozano-M and Nayiver G. Caicedo-D/

\section{Journal of Engineering Science and Technology Review 11 (4) (2018) 180 - 198}

index depends on A, the relationship between voltage at the receiving end and voltage at the sending end, and the phase angle across the line. This suggests that the loadability of a long transmission line decreases due to the decrease in the magnitude of " $A$ ". The network load capacity is limited by the length of a line in the network.

Gu et al., 2009. Gu \& Wan., 2010: in [59] and [60], the LMVSI (Linearized Motor Voltage Stability Index) is presented. It considers the characteristics of the dynamic loads of an induction motor, and is based on the determinant of the equivalent load state matrix. The index demands a WAMS system for its implementation in addition to previous knowledge of the motor parameters. The index does not require calculating the network equivalents which improves the accuracy and the calculation speed of the linearized index, and can be used to predict the load margin.

The composite load model of an induction motor in parallel with a constant impedance is sufficient for the analysis of voltage stability. In the formulation of the index, the third-order model of an induction motor obtained by ignoring the transients of the stator is used. Fig. 9 shows the composite load model of induction motor and constant impedance.

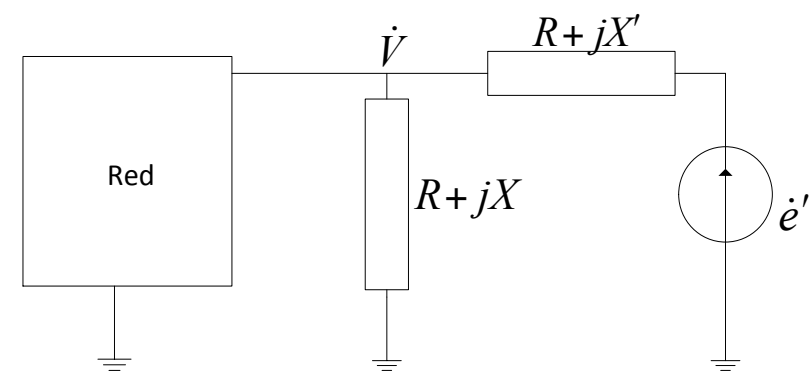

Fig. 9. Composite load model of an induction motor and constant impedance.

In order to determine the local voltage stability index, voltages and powers measured at different times are used. In addition, only local dynamics are considered due to the induction motor. The properties of the 'A' state matrix are used to evaluate the stability of each node.

Generator models, reactive power limits, saturation of the transmission line, etc., they are implicitly taken into account by the measurements obtained from PMUs, and therefore, they are not modeled directly. In a generic time $t_{i}$, the proposed motor voltage stability index (MVSI) is defined in equation

$$
\operatorname{MVSI}_{i, k}=\left|\operatorname{det}\left(A_{i, k}\right)\right|
$$

Where, $A_{i, k}$ is the Jacobian matrix at a given time $t_{i}$ and the node k. $A_{i, k}$ depends on the node voltage and load power obtained through PMU measurements. The MVSI index can be easily linearized by using equation

(91):

$$
L M V S I_{i, k}=\frac{M V S I_{i, k}}{\left|d M V S I_{i, k} / d \lambda_{i, k}\right|}
$$

Here $\lambda_{i, k}$ is the factor of loadability given at time $t_{i}$, and it is calculated by equation

$$
\text { (92): }
$$

$\lambda_{i, k}=\left(P_{L, k}^{t_{i}}-P_{L, k}^{t_{0}}\right) / P_{L, k}^{t_{0}}$
Where $P_{L, k}^{t_{i}}$ and $P_{L, k}^{t_{0}}$ are the active power at instants $t_{i}$ and $t_{0}$ respectively. To calculate the critical load margin of each node (i.e., the value of $\lambda$, for which LMVSI $=0$ ), it is necessary to know how the voltage of the node varies as a function of load powers. In some references this information is obtained by determining the Thevenin equivalent of the network in a load node. However, this step can take a long time, especially for online applications. For this reason, in this article measurements of voltages and powers are used at different times, instead of calculating equivalents. If the load on node $k$ is varied in the time interval between $t_{i}$ and $t_{i-1}$, assuming that the index is completely linear, the predicted critical load margin $\lambda_{k}^{\text {crit }}$ can be expressed by equation (93):

$\lambda_{k}^{c r i t}=\lambda_{i-1, k}-\frac{L M V S I_{i-1, k}\left(\lambda_{i, k}-\lambda_{i-1, k}\right)}{L M V S I_{i, k}-L M V S I_{i-1, k}}$

In order to compute $\lambda_{k}^{c r i t}$, it is necessary that $L M V S I_{i, k}<$ $\operatorname{LMVSI}_{i-1, k}$, that is to say, when load power consumption has increased. The minimum critical load margin expected for all nodes is $\lambda^{\text {crit }}=\min \left\{\lambda_{k}^{\text {crit }}\right\}$. To calculate LMVSI, a measurement in the current time period is enough. Whereas, to calculate $\lambda_{k}^{c r i t}$, two sufficiently different measurements are required in different time periods. First, two VSIs, called $L M V S I_{i, k}$ and $L M V S I_{i-1, k}$, are calculated with equation

(91). Then, the loading margin at node $\mathrm{k}$ is obtained with equation

Parniani \& Vanouni., 2010: another advance to the SDC index is presented in [61], where an index called DSY (Derivate (D) of the load apparent power (S) with respect to its admittance (Y)), which uses only the RMS values of voltage and current, is proposed. The local DSY index estimates the proximity to the loadability limit online, with two consecutive scalar local measurements. This index is based on the fact that the apparent load power variation is zero at loadability limit. The disadvantages of the SDC index are that it requires two consecutive local voltage and current phasor measurements, as well as phasor measuring instruments for its calculation. The other problem with this method (SDC) is that the value of the index is positive in both stable and unstable regions, which corresponds to the upper and lower parts of the PV curve. Therefore, if a large disturbance occurs near the monitored node, resulting in a sudden movement from the stable to the unstable region with a reasonable voltage level, this index cannot distinguish the arrival to the unstable region. These disadvantages were addressed by the DSY index.

Index DSY is calculated by equation (94):

$D S Y=\frac{\Delta S_{i}}{V_{i}^{2} \Delta Y_{i}}=\frac{S_{i, 2}-S_{i, 1}}{\left(V_{i, 1}\right)^{2}\left(\frac{I_{i, 2}}{V_{i, 2}}-\frac{I_{i, 1}}{V_{i, 1}}\right)}$

Since $\mathrm{S}=\mathrm{VI}$, to calculate the index only two consecutive measurements (represented by subscripts 1 and 2) of $I_{i}$ and $V_{i}$, which are readily available scalar local measurements, are needed.

Abasi \& Karbalaei., 2011: in [62], an improvement strategy to the BSDC index considering the voltagedependent load models is presented. At the point of maximum power transfer, the BSDC index is reduced to 0 . Therefore, if it is a voltage independent load, the BSDC 
Sindy L. Ramírez-P, Carlos A. Lozano-M and Nayiver G. Caicedo-D/

Journal of Engineering Science and Technology Review 11 (4) (2018) $180-198$

criterion can detect the voltage collapse point. But, if part of the load is a voltage-dependent type, the voltage stability limit extends beyond the maximum power transfer point, and the BSDC index cannot determine reliably the proximity to voltage instability. The improvement of the BSDC criterion in these conditions (voltage-dependent load) by using the load model given in [62] consists in separating the load into voltage-dependent and voltage-independent parts. Assuming that there is a mixed load at node $i$, the apparent power is calculated by

$\dot{S}_{i}=\dot{V}_{i} \dot{I}_{i}^{*}=\dot{S}_{\text {indepn }}+\dot{S}_{\text {depn }}$

Where $\dot{S}_{\text {indepn }}$ and $\dot{S}_{\text {depn }}$ represent the apparent power from the load voltage independent and dependent parts respectively. The current that is related to the voltage independent part is calculated by equation

(96):

$$
\dot{I}_{\text {indepn }}=\frac{\dot{S}_{\text {indepn }}^{*}}{\dot{V}_{i}^{*}}
$$

To calculate the improved BSDC index using equation (97), the current from equation (96) is used:

$B S D C_{\text {indep }}=1-\left|\frac{\Delta \dot{V}_{i} \dot{I}_{\text {indep } n}^{*}}{\Delta \dot{I}_{\text {indepn }}^{*} \dot{V}_{i}}\right|$

The value of $B S D C_{\text {indep }}$ is reduced to 0 at the point of voltage collapse, regardless of whether the load is voltage dependent or independent because at the point of voltage collapse the term $\dot{S}_{\text {indepn }}$ reaches the maximum value, and does not change by a small alteration in $\dot{I}_{\text {indepn }}$ and $\dot{V}_{i}$. $B S D C_{\text {indep }}$ can be calculated at each node of PES, and when the minimum value of all indices $B S D C_{\text {indep }}$ becomes smaller than a predefined threshold voltage collapse is reached.

Sodhi et al., 2012: later on, another index called SVIMI (Synchrophasor based Voltage Instability Monitoring Index) was developed in [63]. This index uses a node voltage magnitude and its consecutive deviations.

The first voltage stability monitoring criterion considered in [63] is voltage magnitude. To maintain voltage stability, voltage deviation in each load node must be as far as possible from its desired value. Voltage Deviation From its Reference (DFR) or the nominal value in each instantaneous measurement-k is calculated by equation (98):

$$
D F R_{k}=\dot{V}_{r e f}-\dot{V}_{k}
$$

In (98), $\quad \dot{V}_{\text {ref }}$ is the reference voltage value, which corresponds to the nominal voltage level, and $\dot{V}_{k}$ indicates the voltage average value. To calculate the average voltage, a sliding window is used on the past voltage measurements $\mathrm{M}$, and at any instant-k. The average voltage is calculated as $\dot{V}_{k}=\left(\frac{1}{M}\right)\left(V_{k}+V_{k-1}+\cdots+\right.$ $\left.V_{k-M+1}\right)$.

Voltage decreases in the system during the evolution of long-term voltage instability. However, it is also possible for a system to go into unstable voltage state near nominal voltage levels. Therefore, the voltage instability that leads to voltage collapse cannot always be predicted just by monitoring voltage magnitudes. The second criterion used in SVMI that reflects imminent voltage instability is the voltage decay rate. During a constant sampling time $\Delta t$, the difference between voltage at two consecutive time instants indicates the voltage decay rate and, therefore, the consecutive voltage deviation (Consecutive Voltage Deviation CVD) at the instant-k. This can be defined by equation

$C V D_{k}=\dot{V}_{k-1}-\dot{V}_{k}$

As the system moves from a stable to an unstable voltage state, voltage drops and, therefore, the CVD value increases. Generally, at a voltage level of 0.8 p.u. in transmission systems, load shedding is exerted in most systems to avoid voltage instability. The potential benefit of considering the voltage decay rate comes from the fact that stable and collapse cases can be easily distinguished at a higher voltage level.

The two criteria in equations

(98) and

(99) are normalized and combined to define a Voltage Instability Monitoring Index (VIMI), at instant-k, which is calculated in equation (100):

$V I M I_{k}=W_{1}(k) \frac{D F R_{k}}{D F R_{\max }}+W_{2}(k) \frac{C V D_{k}}{C V D_{\max }}$

$W_{1}(k)$ and $W_{2}(k)$ are the weighing factors for the two criteria at the time instant $\mathrm{k}$, so that $W_{1}(k)+W_{2}(k)=1$. In (100), $D F R_{\max }$ and $C V D_{\max }$ are the maximum allowable deviations in the two criteria, and their values are dependent on the system, requiring extensive off-line system studies for the base system as well as the cases of contingency in order to fix their values. A range in which the values of these two parameters are generally close to the point of collapse is obtained. The VIMI, calculated at each load- $l$ node, can finally be used to define SVIMI; it based on synchrophasors of the whole system, which is calculated in equation (101):

$S V I M I_{k}=\max \left(V I M I_{k}^{l}\right) ; l \in$ conjunto de nodos de carga

(101)

The numerator in (100) is the measured deviation, and the denominator is the tolerable deviation in the two criteria under consideration. As the measured deviation approaches the tolerable deviation SVIMI approaches the numerical value 1.0, which indicates a voltage instability condition.

Zheng \& Kezunovic., 2010, 2012 y 2013: in [64], a methodology using synchrophasor measurements taken from PMU is developed for online stability estimation and early detection of impending system instability events by using Decision Trees (DT). In [65], this index was adapted for distribution systems which include different types of wind turbines by using only local measurements in the substation. The VSI index quantifies the voltage stability margin of a general two-node transmission system from PMU data at the two ends of the line. Subsequently, the VSI is modified to adapt to the conditions of the radial distribution system. Only local measurements are needed after the modification; without time synchronization.

From Fig. 6 the index for stability margin in distribution systems is derived. Then, with equation

(102) VSI index is calculated: 
Sindy L. Ramírez-P, Carlos A. Lozano-M and Nayiver G. Caicedo-D/

Journal of Engineering Science and Technology Review 11 (4) (2018) 180 - 198

$V S I=$

$\frac{\left|V_{i}\right|^{2} \sqrt{\left[\left(P_{i}-|I|^{2} r_{i j}\right)^{2}+\left(Q_{i}-|I|^{2} x_{i j}\right)^{2}\right]\left(r_{i j}{ }^{2}+x_{i j}{ }^{2}\right)}}{2\left(P_{i} x_{i j}-Q_{i} r_{i j}\right)^{2}}-$

$\frac{\left|V_{i}\right|^{2}\left[\left(P_{i}-|I|^{2} r_{i j}\right) r_{i j}+\left(Q_{i}-|I|^{2} x_{i j}\right) x_{i j}\right]}{2\left(P_{i} x_{i j}-Q_{i} r_{i j}\right)^{2}}$

Tianjiao Pu et al., 2014: in [66], a fast calculation voltage stability index called $\mathrm{L}_{\mathrm{fci}}$ which is based on the feasible solution of voltage is proposed. This index can be obtained through an algebraic calculation made with the PMU measurement information, and it can be applied online. This index is also derived from a more precise model that considers the lines capacitance. On the other hand, it is shown that $\mathrm{L}_{\mathrm{fci}}$ is still accurate taking into account the voltage characteristics of ZIP loads.

Unlike previous indices that are based on the feasible solution of the system lines power flow equation, $\mathrm{L}_{\mathrm{fci}}$ is obtained from the voltage equation of each line in the system. Fig. 10 shows the equivalent line model with its capacitances.

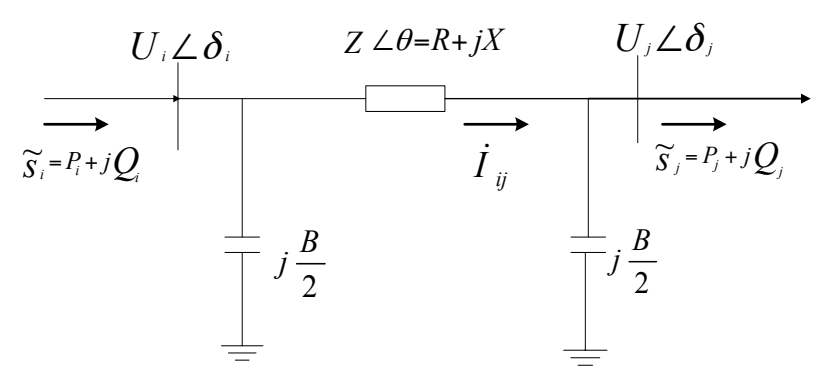

Fig. 10. Equivalent Line Model with capacitance.

Considering Fig. 10, the expression of $\mathrm{L}_{\mathrm{fci}}$ is calculated by equations

(103), (104) and

(105):

$L_{x}=\frac{4\left(1-\frac{B(X-R)}{2}\right)\left[P_{j}(X+R)+Q_{j}(X-R)\right]}{U_{i}^{2}(1+\sin 2 \delta)}<1$

$L_{y}=\frac{4\left(1-\frac{B(X+R)}{2}\right)\left[P_{j}(R-X)+Q_{j}(R+X)\right]}{U_{i}^{2}(1-\sin 2 \delta)}<1$

$L_{f c i}=\max \left\{L_{x}, L_{y}\right\}$

The regional characteristic of voltage stability determines that voltage collapse occurs whenever one of the lines in the system has voltage stability problems. $\mathrm{L}_{\mathrm{fci}}$ index can indicate weak lines of voltage stability in the system. If $\mathrm{L}_{\mathrm{fci}}$ tends to be 1 , the system will be closer to the voltage stability critical point. Therefore, the maximum value of $\mathrm{L}_{\mathrm{fci}}$ for all lines in the system can be used as a global index to indicate voltage stability in the whole system, and it is calculated by the equation

(106):

$L_{f c i, \max }=\max _{j \in R}\left\{L_{f c i}^{(j)}\right\}$

Where $R$ is the number of lines.

Ratra, Tiwari \& Niazi. 2018: in [67], the proposed index is derived from a voltage quadratic equation. The proposed index is based upon ABCD parameters of transmission line, which include line capacitance and resistance that are neglected by existing voltage stability indices. Therefore, the proposed index assesses voltage sensitivity precisely under all the conditions, and indicates the system's proximity to voltage collapse point. Voltage/current at the emitter end of a line can be related to voltage/current of the receiver end by using $\mathrm{ABCD}$ parameters presented in equation

$\left[\begin{array}{l}V_{s} \\ I_{s}\end{array}\right]=\left[\begin{array}{ll}A & B \\ C & D\end{array}\right]\left[\begin{array}{l}V_{R} \\ I_{R}\end{array}\right]$

The terms A, B, $\mathbf{C}$ and $\mathbf{D}$ are known as transmission line parameters. The terms in bold letters are indicative of phasor quantities, while terms in normal letters indicate scalar magnitudes. A, B, C and $\mathbf{D}$ parameters of transmission system are expressed by equations

(108),

(109),

(110) and

$\boldsymbol{A}=1+\boldsymbol{Y} * \frac{Z}{2}$

$\boldsymbol{B}=\boldsymbol{Z}$

$\boldsymbol{C}=\boldsymbol{Y} *\left[1+\boldsymbol{Y} * \frac{\boldsymbol{Z}}{4}\right]$

$\boldsymbol{D}=\boldsymbol{A}$

The proposed LVSI for $\mathrm{j}$-th line can be defined based on equation (112).

$L V S I_{j}=\frac{2 V_{R j} \mathrm{~A}_{j} \cos \left(\beta_{j}-\alpha_{j}\right)}{V_{S j} \cos \left(\beta_{j}-\delta_{S R j}\right)} \forall j=1,2,3 \ldots l$

$L V S I_{j}$ index is calculated for all the lines simultaneously. Without load, the value of LVSI=2. It is to be noted that under no load conditions the system remains mostly stable. Under maximum load conditions, the value of LVSI=1. Therefore, it has been shown mathematically that the value of LVSI varies between 2 and 1, i.e., it goes from the most stable state to voltage collapse. LVSI index identifies critical lines. The voltage stability global indicator for the whole system can be found in equation (113):

$L V S I=\max L_{V S I} \quad \forall j=1,2,3 \ldots l$

To avoid the system voltage collapse, the proposed index must be greater than 1, i.e, $L V S I>1$. The advantage of this approach is that indices of different lines are computed simultaneously. This index is very simple and requires only data from nodes voltage phasors, so it can be easily calculated online.

\section{Analysis of the Bibliographic Review}

The approaches that use PES variables obtained through measurements have advantages associated with the acquisition of data that allow to analyze SP voltage stability in a direct way, before different operating conditions and contingencies, in the nodes as well as the lines. However, these measurement-based approaches also present challenges that are related to the large size of PES nowadays, which leads to the need to have measurement units in strategically located nodes to get complete observability of the system. 
Sindy L. Ramírez-P, Carlos A. Lozano-M and Nayiver G. Caicedo-D/

\section{Journal of Engineering Science and Technology Review 11 (4) (2018) 180 - 198}

Nevertheless, some PES in countries such as Norway, China, India, the United States, Colombia and Brazil currently have this technology [2].

Although PMU technology is increasingly used in PES, according to this bibliographic review there are still very few references that use these tools for voltage stability monitoring with PMU measurements. Another relevant characteristic of this measurement-based approach is the definition of indices involving the dynamic characteristics of loads, which despite of being few, allow analysis to be carried out increasingly closer to PES real conditions. Therefore, online voltage stability monitoring based on synchronized phasor measurements is a work area with great research potential since it allows to use the advantages of modern measurement systems such as WAMS through PMUs, facilitating the access to voltage measurements and current phasors synchronized via GPS (Global Positioning Systems) in many of the nodes.

Fig. 11 presents a classification of voltage stability indices based on measurements and/or parameters, taking as reference the classification given in [2]. In this case, emphasis is placed on indices based on PMU measurements and the identification of node and line indices.

In Table 1, a comparative table to define the characteristics of the indices mentioned in the previous section is presented. The table shows the comparison made between voltage stability indices, in which column I refers to the name of the index, author and year, column II defines whether the index considers the load model in its formulation or the index performance evaluation, column III corresponds to the indices based on PMU measurements, and Column IV refers to the application of the index in real time. Column V indicates whether the index has operational restrictions in a real PES, where number 1 corresponds to the reactive limits of the generator, number 2 to voltage limits for nodes, and number 3 to lines loadability. Column VI indicates in which PES the indices have been implemented. Finally, column VII illustrates whether the index has been applied to large power systems.

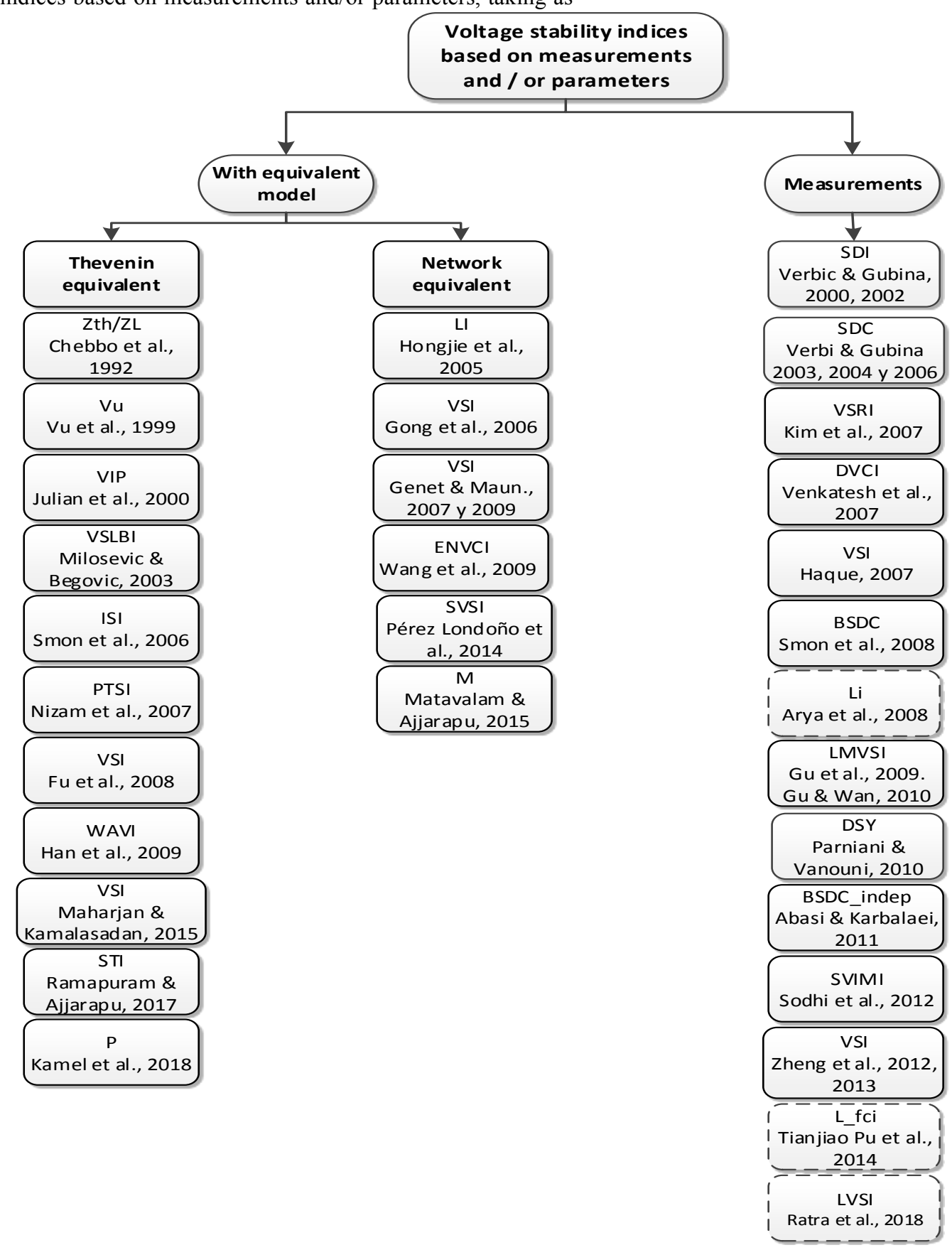

Fig. 11. Classification of indices for voltage stability monitoring based on phasor measurements and/or parameters.

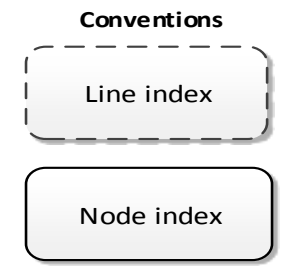


Sindy L. Ramírez-P, Carlos A. Lozano-M and Nayiver G. Caicedo-D/

Journal of Engineering Science and Technology Review 11 (4) (2018) 180 - 198

Table 1. Comparison of the indices of voltage stability based on measurements and/or parameters.

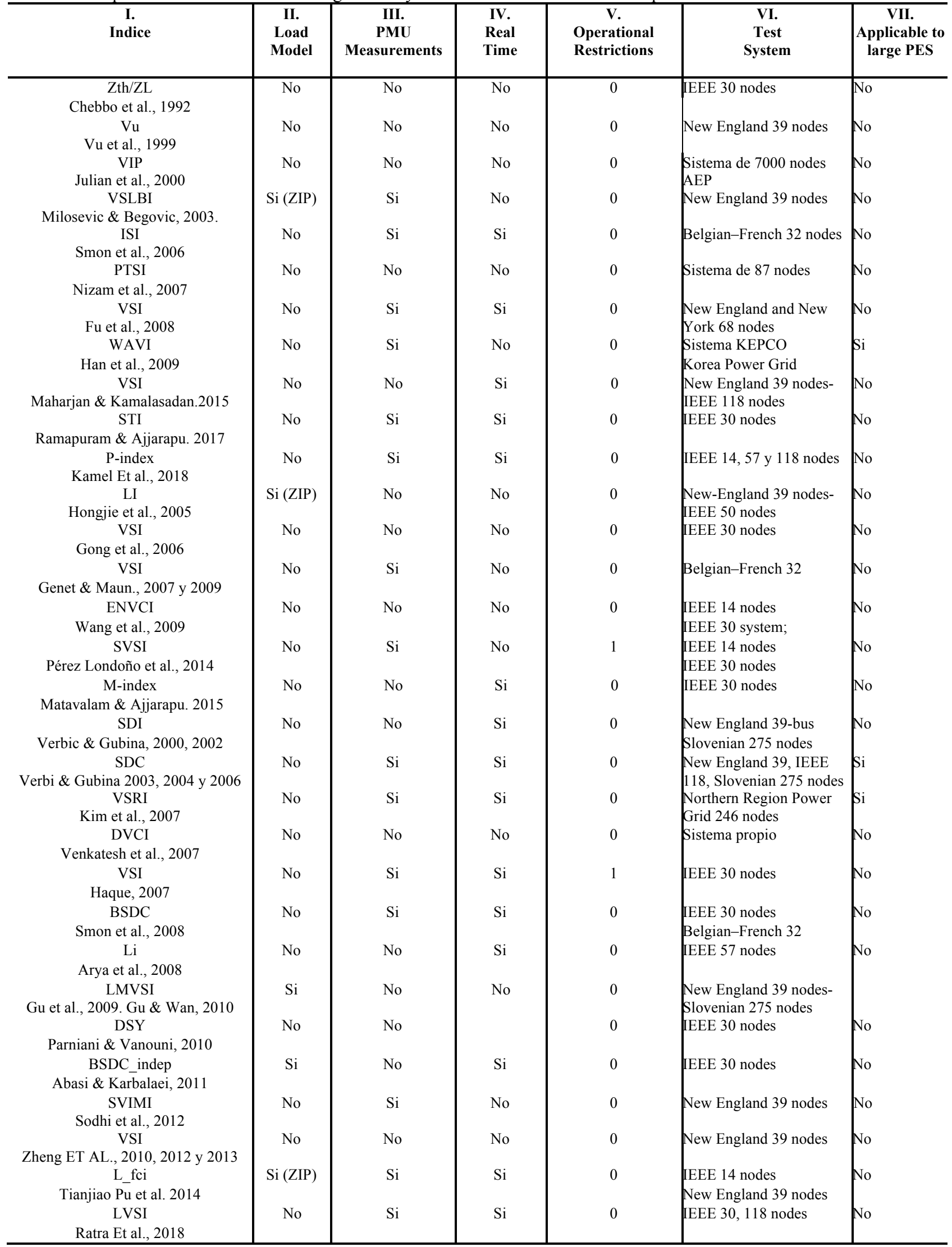

Voltage stability monitoring methods and indices based on the Thevenin equivalent have a correct theoretical base. Since Thevenin equivalent parameters are not easy to follow, some authors propose some voltage stability evaluation methods and indices without identifying Thevenin equivalent parameters. 


\section{Conclusions}

According to this review and analysis, there are some weaknesses in the indices for voltage stability monitoring. Any index is based on considering the voltage levels for transmission according to technical standards. Indices are not used in control strategies to avoid stability problems considering voltage levels and generator limits.

The obtained Thevenin equivalent parameters are based on static stability (only voltage sources and impedances), and power systems nowadays are dynamic in terms of their load, configuration and dispatch. Obtaining Thevenin equivalent parameters is still a very big problem for dynamic power systems.

The estimation of voltage stability margin can be misleading if it is not combined with generator reactive power limitations.
Some SDC and SDI indices are based on the variation between the two consecutive states of the system, in which load variations for the identification of the Thevenin parameters are required. In more realistic cases, PES are constantly changing the power required by the load; therefore, using this criterion can be used reliably.

Very few investigations have evaluated voltage stability using monitoring tools, they neither have included electrical components, such as over-excitation limiters OEL (Over Excitation Limitator) and Tap Changers Under Load (OLTC) (On-Load Tap Changer) which create discrete changes affecting voltage instability.

This is an Open Access article distributed under the terms of the Creative Commons Attribution License

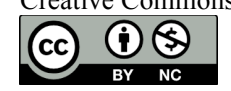

\section{References}

[1] S. M. Pérez Londoño, "Sandra Milena Pérez Londoño," Universidad Nacional de Colombia, 2013.

[2] S. M. Pérez Londoño, G. Olivar Tost, and J. J. Mora Flórez, "Una propuesta de clasificación para los índices de estabilidad de tensión," Ing. y Compet., vol. 130, no. 2, pp. 115-130, 2014.

[3] C. E. D. Cardet, "Analysis on Voltage Stability Indices," RWTH Aachen University, 2012.

[4] M. Cupelli, C. Doig Cardet, and A. Monti, "Voltage stability indices comparison on the IEEE-39 bus system using RTDS," in 2012 IEEE International Conference on Power System Technology (POWERCON), 2012, pp. 1-6.

[5] J. Modarresi, E. Gholipour, and A. Khodabakhshian, "A comprehensive review of the voltage stability indices," Renew. Sustain. Energy Rev., vol. 63, pp. 1-12, 2016.

[6] J. Zhao and Y. Yang, "A review on on-line voltage stability monitoring indices and methods based on local phasor measurements," in 17th Power Systems Computation Conference, 2011, pp. 1-7.

[7] Z. Xi and W. C. Kong, "Comparison of voltage stability indexes considering dynamic load," in 2011 IEEE Electrical Power and Energy Conference, 2011, pp. 249-254.

[8] C. Reis, "A Comparison of Voltage Stability Indices," no. March, pp. 1007-1010, 2006.

[9] P. Rajalakshmi and M. Rathinakumar, "A comparison of transmission line voltage stability indices," Proceeding IEEE - 2nd Int. Conf. Adv. Electr. Electron. Information, Commun. BioInformatics, IEEE - AEEICB 2016, pp. 44-48, 2016.

[10]A. Oukennou, A. Sandali, E. S. Team, and I. Team, "Voltage stability indices sensitivity evaluation under load variation in electrical power system," Electr. Inf. Technol. (ICEIT), 2017 Int. Conf., pp. 1-7, 2017.

[11]A. Oukennou and A. Sandali, "Assessment and analysis of voltage stability indices in electrical network using PSAT Software," in 2016 Eighteenth International Middle East Power Systems Conference (MEPCON), Cairo, 2016, 2016, pp. 705-710.

[12]F. Karbalaei, H. Soleymani, and S. Afsharnia, "A comparison of voltage collapse proximity indicators," in 2010 Conference Proceedings IPEC, 2010, pp. 429-432.

[13]M. Glavic and T. Van Cutsem, "Wide-Area Detection of Voltage Instability From Synchronized Phasor Measurements. Part I: Principle," IEEE Trans. Power Syst., vol. 24, no. 3, pp. 1408-1416, Aug. 2009.

[14]B. Genet, "On monitoring methods and load modeling to improve voltage stability assessment efficiency," Université Libre de Bruxelles (U.L.B.), 2009.

[15]P. Kundur, Power System Stability and Control, McGraw-Hil. New York, USA, 1994.

[16]P.-A. Lof, T. Smed, G. Andersson, and D. J. Hill, "Fast calculation of a voltage stability index," IEEE Trans. Power Syst., vol. 7, no. 1, pp. 54-64, 1992.

[17]B. Gao, G. K. Morison, and P. Kundur, "Voltage stability evaluation using modal analysis," IEEE Trans. Power Syst., vol. 7, no. 4, pp. 1529-1542, 1992.
[18]C. A. Cañizares, "Voltage Stability Assessment: Concepts , Practices and Tools," in IEEE-PES Power System Stability Subcommittee Special Publication, 2002, no. August.

[19]L. F. Rodriguez García, "Análisis de estabilidad de tensión considerando sistemas de monitoreo de área amplia y características de cargas mixtas," Universidad Tecnológica de Pereira, 2014.

[20]V. Terzija et al., "Wide-Area Monitoring, Protection, and Control of Future Electric Power Networks," Proc. IEEE, vol. 99, no. 1, pp. 80-93, Jan. 2011.

[21]O. Nelles, Nonlinear System Identification. Berlin, Heidelberg: Springer Berlin Heidelberg, 2001.

[22] J. R. Raol, G. Girija, and J. Singh, Modelling and Parameter Estimation of Dynamic Systems. The Institution of Engineering and Technology, Michael Faraday House, Six Hills Way, Stevenage SG1 2AY, UK: IET, 2004.

[23] A. Chebbo, M. R. Irving, and M. J. H. Sterling, "Voltage collapse proximity indicator: behaviour and implications," in Generation, Transmission and Distribution, IEE Proceedings C, 1992, vol. 139, no. 3, pp. 241-252.

[24]K. Vu, M. M. Begovic, D. Novosel, and M. M. Saha, "Use of local measurements to estimate voltage-stability margin," IEEE Trans. Power Syst., vol. 14, no. 3, pp. 1029-1035, 1999.

[25]D. E. Julian, R. P. Schulz, K. T. Vu, W. H. Quaintance, N. B. Bhatt, and D. Novosel, "Quantifying proximity to voltage collapse using the Voltage Instability Predictor (VIP)," in 2000 Power Engineering Society Summer Meeting (Cat. No.00CH37134), 2000, vol. 2, pp. 931-936.

[26]B. Milosevic and M. Begovic, "Voltage-stability protection and control using a wide-area network of phasor measurements," IEEE Trans. Power Syst., vol. 18, no. 1, pp. 121-127, Feb. 2003.

[27] I. Smon, G. Verbic, and F. Gubina, "Local Voltage-Stability Index Using Tellegen's Theorem," IEEE Trans. Power Syst., vol. 21, no. 3, pp. 1267-1275, Aug. 2006.

[28]M. Nizam, A. Mohamed, and A. Hussain, "Dynamic Voltage Collapse Prediction on a Practical Power System Using Power Transfer Stability Index," in 2007 5th Student Conference on Research and Development, 2007, pp. 1-6.

[29] L. Fu, B. C. Pal, and B. J. Cory, "Phasor measurement application for power system voltage stability monitoring," in 2008 IEEE Power and Energy Society General Meeting - Conversion and Delivery of Electrical Energy in the 21st Century, 2008, pp. 1-8.

[30] S. Han, B. Lee, S. Kim, and Y. Moon, "Development of voltage stability index using synchro-phasor based data," in 2009 Transmission \& Distribution Conference \& Exposition: Asia and Pacific, 2009, pp. 1-4.

[31]S. Han, B. Lee, S. Kim, and Y. Moon, "Real Time Wide Area Voltage Stability Index in the Korean Metropolitan Area," JEET, vol. 4, no. 4, pp. 451-456, 2009.

[32]M. Larsson, C. Rehtanz, and J. Bertsch, "Monitoring and operation of transmission corridors," in 2003 IEEE Bologna Power Tech Conference Proceedings, 2003, vol. 3, pp. 690-697.

[33]M. Larsson, C. Rehtanz, and J. Bertsch, "Real-Time Voltage Stability Assessment Of Transmission Corridors," in IFAC Power 
Sindy L. Ramírez-P, Carlos A. Lozano-M and Nayiver G. Caicedo-D/

\section{Journal of Engineering Science and Technology Review 11 (4) (2018) 180 - 198}

Plants \& Power Systems Control, 2003, pp. 1-6.

[34]M. Zima, M. Larsson, P. Korba, C. Rehtanz, and G. Andersson, "Design Aspects for Wide-Area Monitoring and Control Systems," Proc. IEEE, vol. 93, no. 5, pp. 980-996, May 2005.

[35]R. Maharjan and S. Kamalasadan, "Voltage Stability Index for Online Voltage Stability Assessment," North Am. Power Symp., 2015.

[36]A. R. Ramapuram Matavalam; V. Ajjarapu, "Validation of the Sensitivity based Thevenin Index on Large Systems," in 2017 IEEE Power \& Energy Society General Meeting, Chicago, IL, 2017, 2017, no. 2, pp. 1-5.

[37]A. R. Ramapuram Matavalam; V. Ajjarapu, "Sensitivity based Thevenin Index with Systematic Inclusion of Reactive Power Limits," Ieee Trans. Power Syst., vol. 33, no. 1, p. 1, 2017.

[38]M. M. M. Kamel, A. A. Karrar, and A. H. Eltom, "Development and Application of a New Voltage Stability Index for On-Line Monitoring and Shedding," IEEE Trans. Power Syst., vol. 33, no. 2, pp. 1231-1241, 2018.

[39]J. Hongjie, Y. Xiaodan, and Y. Yixin, "An improved voltage stability index and its application," Int. J. Electr. Power Energy Syst., vol. 27, no. 8, pp. 567-574, Oct. 2005.

[40]P. Kessel and H. Glavitsch, "Estimating the Voltage Stability of a Power System," IEEE Trans. Power Deliv., vol. 1, no. 3, pp. 346354, 1986.

[41]Y. Gong, N. Schulz, and A. Guzmán, "Synchrophasor-Based RealTime Voltage Stability Index," in 2006 IEEE PES Power Systems Conference and Exposition, 2006, pp. 1029-1036.

[42] V. Salehi, A. Mazloomzadeh, and O. Mohammed, "Development and Implementation of a Phasor Measurement Unit for Real-Time Monitoring, Control and Protection of Power Systems," in 2011 IEEE, Power and Energy Society General Meeting, 2011, pp. 1-7.

[43]B. Genet and J.-C. Maun, "Voltage-Stability Monitoring Using Wide-Area Measurement Systems," in 2007 IEEE Lausanne Power Tech, 2007, pp. 1712-1717.

[44]Y. Wang, W. Li, and J. Lu, "A new node voltage stability index based on local voltage phasors," Electr. Power Syst. Res., vol. 79, no. 1, pp. 265-271, Jan. 2009.

[45] S. Pérez-Londoño, L. F. Rodríguez, and G. Olivar, "A Simplified Voltage Stability Index (SVSI)," Int. J. Electr. Power Energy Syst., vol. 63, pp. 806-813, Dec. 2014.

[46] A. R. R. Matavalam and V. Ajjarapu, "Calculating the long term voltage stability margin using a linear index," IEEE Power Energy Soc. Gen. Meet., vol. 2015-Septe, no. 2, 2015.

[47]G. Verbi and F. Gubina, "Fast voltage-collapse line-protection algorithm based on local phasors," IEE Proc. - Gener. Transm. Distrib., vol. 150, no. 4, pp. 482-486, 2003.

[48] G. Verbic and F. Gubina, "A new concept of protection against voltage collapse based on local phasors," in PowerCon 2000. 2000 International Conference on Power System Technology. Proceedings (Cat. No.00EX409), 2000, vol. 2, pp. 965-970.

[49] G. Verbic and F. Gubina, "Fast algorithm for voltage collapse protection based on local phasors," in IEEE Power Engineering Society Summer Meeting, 2002, vol. 3, pp. 1650-1655.

[50]G. Verbic and F. Gubina, "A novel concept for voltage collapse protection based on local phasors," in IEEE/PES Transmission and Distribution Conference and Exhibition, 2002, vol. 1, pp. 124-129.
[51]G. Verbic and F. Gubina, “A New Concept of Voltage-Collapse Protection Based on Local Phasors," IEEE Trans. Power Deliv., vol. 19, no. 2, pp. 576-581, Apr. 2004.

[52] G. Verbič, M. Pantoš, and F. Gubina, "On voltage collapse and apparent-power losses," Electr. Power Syst. Res., vol. 76, no. 9-10, pp. 760-767, Jun. 2006.

[53]C. J. Zapata, M. Ríos, and O. Gómez, "Índices de seguridad de voltaje calculados con datos de unidades fasoriales de medida," Rev. Ing. Univ. los Andes. Bogotá, Colomb., pp. 49-55, 2009.

[54]D. J. Kim, Y. H. Moon, H. Y. Kim, and J. Y. Yoon, "System and method for calculating real-time voltage stability risk index in power system using time series data," Patent No. US 7236898 B2, 2007.

[55]B. Venkatesh, A. Rost, and L. Chang, "Dynamic Voltage Collapse Index\&mdash; Wind Generator Application," IEEE Trans. Power Deliv., vol. 22, no. 1, pp. 90-94, Jan. 2007.

[56] M. H. Haque, "Use of local information to determine the distance to voltage collapse," in The 8th International Power Engineering Conference (IPEC 2007), 2007, pp. 407-412.

[57]I. Šmon, M. Pantoš, and F. Gubina, “An improved voltage-collapse protection algorithm based on local phasors," Electr. Power Syst. Res., vol. 78, no. 3, pp. 434-440, Mar. 2008.

[58]L. D. Arya, S. C. Choube, and M. Shrivastava, "Technique for voltage stability assessment using newly developed line voltage stability index," Energy Convers. Manag., vol. 49, no. 2, pp. 267275, Feb. 2008.

[59] W. Gu, Q. Wan, and P. Jiang, "Linearized local voltage stability index considering induction motor load," in 2009 IEEE Power \& Energy Society General Meeting, 2009, pp. 1-5.

$[60]$ W. Gu and Q. Wan, "Linearized voltage stability index for widearea voltage monitoring and control," Int. J. Electr. Power Energy Syst., vol. 32, no. 4, pp. 333-336, May 2010.

[61]M. Parniani and M. Vanouni, "A Fast Local Index for Online Estimation of Closeness to Loadability Limit," IEEE Trans. Power Syst., vol. 25, no. 1, pp. 584-585, Feb. 2010.

[62] S. Abasi and F. Karbalaei, "Diagnosis of Voltage Instability Using BSDC Index in the Presence of Voltage Dependent Loads," in 2011 Asia-Pacific Power and Energy Engineering Conference, 2011, pp. $1-6$.

[63] R. Sodhi, S. C. Srivastava, and S. N. Singh, "A Simple Scheme for Wide Area Detection of Impending Voltage Instability," IEEE Trans. Smart Grid, vol. 3, no. 2, pp. 818-827, Jun. 2012.

[64]C. Zheng, "Power system online stability assessment using synchrophasor data mining," Texas A\&M University, 2013.

[65]C. Zheng and M. Kezunovic, "Distribution system voltage stability analysis with wind farms integration," in North American Power Symposium 2010, 2010, pp. 1-6.

[66] Tianjiao Pu, Zhao Zhang, Ting Yu, Wei Han, and Lei Dong, “A fast calculation static voltage stability index based on wide area measurement system," in 2014 International Conference on Power System Technology, 2014, pp. 30-35.

[67] S. Ratra, R. Tiwari, and K. R. Niazi, "Voltage stability assessment in power systems using line voltage stability index," Comput. Electr. Eng., vol. 0, pp. 1-13, 2018. 